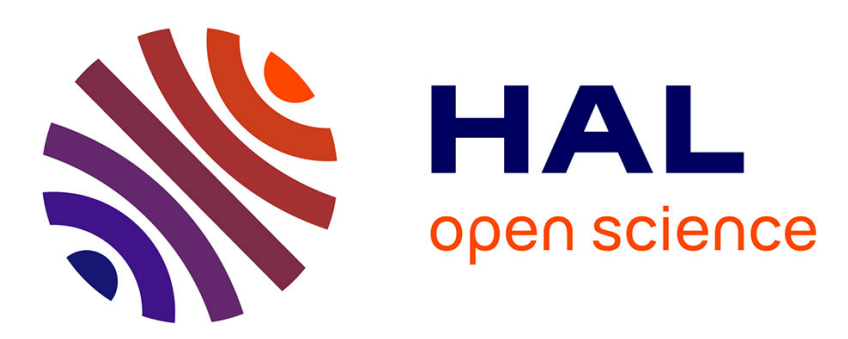

\title{
Nonlinear equations with unbounded heat conduction and integrable data
}

\author{
Dominique Blanchard, Olivier Guibé, Hicham Redwane
}

\section{To cite this version:}

Dominique Blanchard, Olivier Guibé, Hicham Redwane. Nonlinear equations with unbounded heat conduction and integrable data. 2006. hal-00112599

\section{HAL Id: hal-00112599 \\ https://hal.science/hal-00112599}

Preprint submitted on 10 Nov 2006

HAL is a multi-disciplinary open access archive for the deposit and dissemination of scientific research documents, whether they are published or not. The documents may come from teaching and research institutions in France or abroad, or from public or private research centers.
L'archive ouverte pluridisciplinaire HAL, est destinée au dépôt et à la diffusion de documents scientifiques de niveau recherche, publiés ou non, émanant des établissements d'enseignement et de recherche français ou étrangers, des laboratoires publics ou privés. 


\title{
NONLINEAR EQUATIONS WITH UNBOUNDED HEAT CONDUCTION AND INTEGRABLE DATA
}

\author{
Dominique Blanchard ${ }^{(1)(2)}$, Olivier Guibé( ${ }^{(2)}$ and Hicham Redwane ${ }^{(3)}$
}

\begin{abstract}
We consider a class of quasi-linear diffusion problems involving a matrix $\mathbf{A}(t, x, u)$ which blows up for a finite value $m$ of the unknown $u$. Stationary and evolution equations are studied for $L^{1}$ data. We focus on the case where the solution $u$ can reach the value $m$. For such problems we introduce a notion of renormalized solutions and we prove the existence of such solutions.
\end{abstract}

KEYWORDS: nonlinear equations, blowing-up heat conduction, existence, renormalized solutions, integrable data.

RÉSumÉ. Nous considérons une classe de problèmes de diffusion quasi-linéaires pour des matrices $\mathbf{A}(t, x, u)$ qui explosent pour une valeur $m$ finie de l'inconnue $u$. Les cas stationnaire et d'évolution sont traités pour des données intégrables et pour des solutions qui atteignent la valeur $m$. Nous donnons une formulation de solutions renormalisées pour ces problèmes et nous démontrons l'existence de telles solutions.

Mots ClEFs : équations non linéaires, diffusion singulière, existence, solutions renormalisées, donnée intégrable.

\footnotetext{
${ }^{(1)}$ Laboratoire Jacques-Louis Lions, Université Paris VI, Boîte courrier 187, 75252 Paris cedex 05

${ }^{(2)}$ Laboratoire de Mathématiques Raphaël Salem UMR 6085 CNRS - Université de Rouen, Avenue de l'Université, BP.12 76801 Saint-Étienne du Rouvray (France), \{Dominique.Blanchard, Olivier.Guibe\}@univ-rouen.fr

${ }^{(3)}$ Faculté des Sciences Juridiques, Économiques et Sociales, Université Hassan 1, B.P 784, Settat (Maroc), Redwane_Hicham@yahoo.fr
} 


\title{
NONLINEAR EQUATIONS WITH UNBOUNDED HEAT CONDUCTION AND INTEGRABLE DATA
}

\author{
Dominique Blanchard, Olivier Guibé and Hicham Redwane
}

\subsection{IntRoduction}

We investigate a class of diffusion problems, in the stationary and evolution cases, with singular matrices with respect to the unknown. More precisely let $\Omega$ be a bounded domain of $\mathbb{R}^{N}, m$ and $T$ be two positive real numbers and $\beta$ and $\gamma$ be two functions of $\mathcal{C}^{0}((-\infty, m))$ such that $\beta(s) \leq \gamma(s), \lim _{s \rightarrow m^{-}} \beta(s)=+\infty$ and $\int_{0}^{m} \gamma(s) \mathrm{d} s<+\infty$. We consider a Carathéodory matrix field $\mathbf{A}(t, x, s)$ defined on $(0, T) \times \Omega \times(-\infty, m)$ such that

$$
\beta(s)|\xi|^{2} \leq \mathbf{A}(t, x, s) \xi \cdot \xi \leq \gamma(s)|\xi|^{2}
$$

for any $s \in(-\infty, m)$ and any $\xi \in \mathbb{R}^{N}$, almost everywhere in $(0, T) \times \Omega$.

Then the matrix $\mathbf{A}(t, x, s)$ blows up (uniformly with respect to $(t, x))$ as $s \rightarrow m^{-}$. We are interested in the diffusion problems governed by the matrix field $\mathbf{A}(t, x, u)$ in the stationary (A is then independent of $t$ ) or evolution cases (see equations (1.1) and (2.1)). When dealing such problems, the main difficulty is indeed to give a sense to the flux $\mathbf{A}(t, x, u) D u$ on the set $\{(t, x) ; u(t, x)=m\}$, or more precisely to describe the behavior of the energy $\mathbf{A}^{\varepsilon}\left(t, x, u^{\varepsilon}\right) D u^{\varepsilon} \cdot D u^{\varepsilon}$ for approximate solutions $u^{\varepsilon}$. In the elliptic case where the matrix is independent of the space variable and with a diagonal blowing part the analysis was carried out in [6] for $L^{2}$ data where an $L^{2}$ estimate for $\mathbf{A}^{\varepsilon}\left(u^{\varepsilon}\right) D u^{\varepsilon}$ was derived. Then the authors gave two formulations of problem of type (1.1), both of them using a sort of decoupling behavior of the solution on the subset $\{u<m\}$ and on the subset $\{u=m\}$. Indeed another type of assumptions can be adopted when $\mathbf{A}$ depends on the $x$-variable and is not diagonal as in $[8,9,14]$ where one assumes that $\int_{0}^{m} \beta(s) \mathrm{d} s=+\infty$, which forces the solution to avoid the value $m$. Then it is easy to construct a solution $u$ which is strictly less that $m$ almost everywhere in $(0, T) \times \Omega$ (that is meas $\{u=m\}=0)$ (see also Section 6) and to show, at least for $L^{2}$ data, that $\mathbf{A}^{\varepsilon}\left(t, x, u^{\varepsilon}\right) D u^{\varepsilon} \cdot D u^{\varepsilon} \rightarrow \mathbf{A}(t, x, u) D u \cdot D u$ in $L^{1}((0, T) \times \Omega)$. Indeed this is due to the fact that no energy can concentrate on the set $\{u=m\}$. For $L^{1}$ data, the same results hold true with $u^{\varepsilon}$ and $u$ replaced by any truncation $T_{k}\left(u^{\varepsilon}\right)$ and $T_{k}(u)$ (see the definition of $T_{k}$ in Section 1.1).

In the present paper, we focus on the case where $\mathbf{A}(t, x, u)$ is not diagonal, $\int_{0}^{m} \gamma(s) \mathrm{d} s<$ $+\infty$ and for $L^{1}$ data both for elliptic and parabolic problems. If we assume that if $f$ is small in $L^{q}$ for some appropriate $q$ then one can prove that $u<m$ almost everywhere (see [14] for the elliptic case). Here we just assume that $f$ is in $L^{1}$ and then we have to handle the set $\{u=m\}$. We give a notion of renormalized solutions (which is more precise in the elliptic case than the one given in [6]) and we prove the existence of such solutions for $L^{1}$ data. The formulation of the problem consists (as in [6]) in considering the equation on the subset $\{u<m\}$ on the one hand and in specifying the behavior of the energy near the subset 
$\{u=m\}$ where the coefficients are singular on the other hand. For this point of view, it is at least formally similar to the formulation for elliptic problems with measure data considered in [7], where one distinguishes the equation where the measure is smooth and the behavior of the energy where the measure is singular.

Let us point out that the energy condition that we obtain in the present paper is more precise that the one stated in [6].

Let us also emphasize that another difficulty arising in the analysis of diffusion problems with matrices satisfying (0.1) is the possible different behaviors of the functions $\beta$ and $\gamma$ near $m$. Loosely speaking, the lower bound in (0.1) and the equation lead to estimates on $D\left(\int_{0}^{u} \beta(s) \mathrm{d} s\right.$ ) in some $L^{p}$ (depending on the smoothness of $f$ ) while the upper bound of (0.1) naturally yields an estimate on the flux $\mathbf{A}(t, x, u) D u$ if one have an estimate on $D\left(\int_{0}^{u} \gamma(s) \mathrm{d} s\right)$.

The paper is organized as follows. In Part 1 we investigate the elliptic case. In Section 1.1 we precise the assumptions on the data and we give the definition of a solution. Section 1.2 we state the existence theorem and we detail the proof in 3 steps. Part 2 is devoted to the parabolic case. The definition of the solution is given in Section 2.1. Section 2.2 gives the existence theorem together with its proof. Section 2.3 is devoted to concluding remarks concerning the case $\int_{0}^{m} \beta(s) \mathrm{d} s=+\infty$ and to a partial uniqueness result.

\section{Part 1. The elliptic case}

\subsection{Assumption on the data And Definition of a SOlution}

Let $\Omega$ be a bounded domain of $\mathbb{R}(N \geq 1)$ and $m$ be a positive real number. We consider the following nonlinear problem

$$
\left\{\begin{aligned}
-\operatorname{div}(\mathbf{A}(x, u) D u) & =f & & \text { in } \Omega ; \\
u & =0 & & \text { on } \partial \Omega,
\end{aligned}\right.
$$

where the assumptions on the data are detailed below. We assume that

$$
\begin{gathered}
f \in L^{1}(\Omega) \\
\mathbf{A}:(x, s) \mapsto \mathbf{A}(x, s)
\end{gathered}
$$

is a Carathéodory function from $\Omega \times(-\infty, m)$ into $\mathbb{R}_{S}^{N \times N}$, the set of $N \times N$ symmetric matrices, such that there exist two positive function $\beta$ and $\gamma$ in $\mathcal{C}^{0}((-\infty, m))$ which satisfy

$$
\begin{gathered}
\lim _{s \rightarrow m^{-}} \beta(s)=+\infty ; \quad \beta(s) \geq \alpha>0 \quad \forall s \in(-\infty, m) \\
\int_{0}^{m} \gamma(s) \mathrm{d} s<+\infty \\
\forall s \in(-\infty, m), \quad \forall \xi \in \mathbb{R}^{N} \quad \beta(s)|\xi|^{2} \leq \mathbf{A}(x, s) \xi \cdot \xi \leq \gamma(s)|\xi|^{2} \quad \text { a.e. in } \Omega .
\end{gathered}
$$

Remark 1.1. Indeed (1.4)-(1.6) imply that $\lim _{s \rightarrow m^{-}} \gamma(s)=+\infty$ and $\int_{0}^{m} \beta(s) \mathrm{d} s<+\infty$. The study of (1.1) under the assumption $\int_{0}^{m} \beta(s) \mathrm{d} s=+\infty$ is easier (see [10,14] and Section 2.3 because one can then show that there exists a solution such that $u<m$ a.e. in $\Omega$.

Assumptions (1.4), (1.6) imply that the $(x, s)$-dependent norm $\left|\mathbf{A}^{1 / 2}(x, s) \xi\right|$ on $\mathbb{R}^{N}$ blows up as $s$ tends to $m$ uniformly with respect to $x$ in $\Omega$. 
The following notations will be used throughout the paper: for any $k \geq 0$, the truncation at height $k$ is defined by $T_{k}(s)=\max (-k, \min (s, k))$; for any integer $n \geq 1$ and any positive real number $\varepsilon>0$, the functions $\theta_{n}, h_{n}, S_{n}$ and $b_{\varepsilon}$ are defined by

$$
\begin{gathered}
\theta_{n}(s)=\frac{1}{n}\left(T_{n}\left(s-T_{n}(s)\right), \quad h_{n}(s)=1-\left|\theta_{n}(s)\right|,\right. \\
S_{n}(s)=\int_{0}^{s} h_{n}(r) \mathrm{d} r \\
b_{\varepsilon}(r)= \begin{cases}1 & \text { if } r \leq m-2 \varepsilon, \\
1-(r-m+\varepsilon) & \text { if } m-2 \varepsilon \leq r \leq m-\varepsilon, \\
0 & \text { if } r \geq m-\varepsilon .\end{cases}
\end{gathered}
$$

1.1.1. Definition of a solution. In this subsection, we give the definition of a solution of (1.1). This definition is more precise than the one used in [6] in the the sense that it localizes the behavior of the energy near the zone where a solution may reach the value $m$ (see [6]).

Definition 1.2. A measurable function $u$ defined on $\Omega$ is a renormalized solution of (1.1) if

$$
\begin{gathered}
\forall k \geq 0 ; \quad T_{k}(u) \in H_{0}^{1}(\Omega), \\
u \leq m \text { a.e. in } \Omega, \\
\forall k \geq 0 ; \quad \mathbb{1}_{\{-k<u<m\}} \mathbf{A}(x, u) D u \in\left(L^{2}(\Omega)\right)^{N}, \\
\lim _{n \rightarrow+\infty} \frac{1}{n} \int_{\{-2 n<u<-n\}} \mathbf{A}(x, u) D u \cdot D u \mathrm{~d} x=0,
\end{gathered}
$$

for any function $\varphi \in L^{\infty}(\Omega) \cap H^{1}(\Omega)$ such that $D \varphi=0$ a.e. on $\{x \in \Omega ; u(x)=m\}$ one has

$$
\lim _{n \rightarrow+\infty} n \int_{\{m-2 / n<u<m-1 / n\}} \mathbf{A}(x, u) D u \cdot D u \varphi \mathrm{d} x=\int_{\{u=m\}} f \varphi \mathrm{d} x,
$$

for any function $h \in W^{1, \infty}(\mathbb{R})$ such that $\operatorname{supp}(h)$ is compact and $h(m)=0$, $u$ satisfies

$$
-\operatorname{div}[h(u) \mathbf{A}(x, u) D u]+h^{\prime}(u) \mathbf{A}(x, u) D u \cdot D u=h(u) f \quad \text { in } \mathcal{D}^{\prime}(\Omega) .
$$

Remark 1.3 (Comments on Definition 1.2). Conditions (1.10) and (1.13) are classical when dealing with renormalized solutions for partial differential equations with $L^{1}$ data (see [12, $13,2])$. The fact that $u \leq m$ almost everywhere in $\Omega$ is already explained (and is natural) in [6]. By contrast, the condition (1.14) on the behavior of the energy near the subset $\{x \in \Omega ; u(x)=m\}$ is an improvement (even in the case $f \in L^{2}(\Omega)$ ) of the one obtained in [6] where it is written for $\varphi \equiv 1$. As mentioned in the introduction this type of condition on the behavior of the energy near level set of $u$ is also considered in [7].

The condition (1.12), which was established in [6] for a diagonal matrix $\mathbf{A}$, is here strongly linked to the fact that $\int_{0}^{m} \gamma(s) \mathrm{d} s<+\infty$. The equation (1.15) and the conditions on the function $h$ are the same as in [6] to which we refer to claim that every term makes sense in this equation. Let us first recall that since $u \leq m$ almost everywhere in $\Omega$, the condition $h(m)=0$ in (1.15) may be equivalently replaced by $h(r)=0, \forall r \geq m$. 


\subsection{EXISTEnCE RESUlT}

We establish the following theorem.

Theorem 1.4. Under the assumptions (1.2)-(1.6), there exists at least a solution of (1.1) in the sense of Definition 1.2.

Proof. The main difficulty comes from the fact that the functions $\beta$ and $\gamma$ entering in the lower and upper bounds of (1.6) may have different growths when $s$ tends to $m$. Loosely speaking, even if $f \in L^{2}(\Omega)$, (and formally) if one uses $\int_{0}^{u} \beta(s) \mathrm{d} s$ as a test function in (1.1) the lower bound in (1.6) leads to an estimate on $\int_{0}^{u} \beta(s) \mathrm{d} s$ in $H_{0}^{1}(\Omega)$. The upper bound in (1.6) does not permit to obtain any kind of estimates on the fields $\mathbf{A}(x, u) D u \mathbb{1}_{\{u<m\}}$ if no assumption on the relative behavior of $\gamma$ with respect to $\beta$ near $m$ is adopted.

As shown in the sequel, we will use a test function which mixes the two functions $\beta$ and $\gamma$. In some sense, this forces to introduce a specific regularization of $\mathbf{A}(x, s)$ which is different from the one used in [6] (namely a truncation of $\mathbf{A}(x, s)$ near $m$ ).

For any $\varepsilon>0$, we consider the field of matrices $\mathbf{A}^{\varepsilon}(x, s)$ defined on $\Omega \times \mathbb{R}$ by

$$
\mathbf{A}^{\varepsilon}(x, s)=b_{\varepsilon}(s) \mathbf{A}(x, s)+\left(1-b_{\varepsilon}(s)\right) \beta(m-\varepsilon) I,
$$

where $b_{\varepsilon}$ is the function defined in (1.9) and $I$ is the identity matrix of $\mathbb{R}^{N \times N}$. Indeed (1.16) we use the convention

$$
b_{\varepsilon}(s) \mathbf{A}(x, s)=0 \quad \text { for } s \geq m-\varepsilon .
$$

Due to assumptions (1.4) and (1.6), we have

$$
\forall s \in \mathbb{R} ; \quad \forall \xi \in \mathbb{R}^{N} \quad \alpha|\xi|^{2} \leq \mathbf{A}^{\varepsilon}(x, s) \xi \cdot \xi \leq\left(b_{\varepsilon}(s) \gamma(s)+\max _{r \in(0, m-\varepsilon)} \beta(r)\right)|\xi|^{2} .
$$

Using classical result on renormalized solutions for quasi-linear elliptic problems (see e.g. $[7,12,13])$ and $(1.17)$, the following regularized problem admits at least one solution $u^{\varepsilon}$

$$
\left\{\begin{aligned}
-\operatorname{div}\left(\mathbf{A}^{\varepsilon}\left(x, u^{\varepsilon}\right) D u^{\varepsilon}\right) & =f & & \text { in } \Omega ; \\
u^{\varepsilon} & =0 & & \text { on } \partial \Omega .
\end{aligned}\right.
$$

Recall that the sequence $u^{\varepsilon}$ satisfies the following properties (see again $[7,12,13]$ )

$$
\begin{gathered}
T_{k}\left(u^{\varepsilon}\right) \in H_{0}^{1}(\Omega), \quad \forall k \geq 0, \\
\lim _{n \rightarrow+\infty} \frac{1}{n} \int_{\left\{n<\left|u^{\varepsilon}\right|<2 n\right\}} \mathbf{A}^{\varepsilon}\left(x, u^{\varepsilon}\right) D u^{\varepsilon} \cdot D u^{\varepsilon} \mathrm{d} x=0,
\end{gathered}
$$

for any function $h \in W^{1, \infty}(\mathbb{R})$ such that $\operatorname{supp}(h)$ is compact

$$
-\operatorname{div}\left[h\left(u^{\varepsilon}\right) \mathbf{A}^{\varepsilon}\left(x, u^{\varepsilon}\right) D u^{\varepsilon}\right]+h^{\prime}\left(u^{\varepsilon}\right) \mathbf{A}\left(x, u^{\varepsilon}\right) D u^{\varepsilon} \cdot D u^{\varepsilon}=h\left(u^{\varepsilon}\right) f \quad \text { in } \mathcal{D}^{\prime}(\Omega) .
$$

In order to show that, for a subsequence still indexed by $\varepsilon, u^{\varepsilon}$ converges to a solution $u$ of the problem in the sense of Definition 1.2, we first introduce the two sequences of auxiliary functions

$$
v^{\varepsilon}=\int_{0}^{\left(u^{\varepsilon}\right)^{+}}\left(\gamma(s) b_{\varepsilon}(s)+\left(1-b_{\varepsilon}(s)\right) \beta(m-\varepsilon)\right) \mathrm{d} s
$$

and

$$
w^{\varepsilon}=\int_{0}^{\left(u^{\varepsilon}\right)^{+}}\left(\beta(s) b_{\varepsilon}(s)+\left(1-b_{\varepsilon}(s)\right) \beta(m-\varepsilon)\right) \mathrm{d} s .
$$


Remark that, since $\gamma(s) \geq \beta(s) \geq \alpha$ for any $s \in(-\infty, m)$, we have

$$
\alpha\left(u^{\varepsilon}\right)^{+} \leq w^{\varepsilon} \leq v^{\varepsilon} \quad \text { a.e. in } \Omega
$$

and

$$
v^{\varepsilon} \leq \max _{s \in(0, m-\varepsilon)} \gamma(s)\left(u^{\varepsilon}\right)^{+} \text {a.e. in } \Omega \text {. }
$$

Then because of (1.19), $v^{\varepsilon}$ and $w^{\varepsilon}$ satisfy $T_{k}\left(v^{\varepsilon}\right) \in H_{0}^{1}(\Omega)$ and $T_{k}\left(w^{\varepsilon}\right) \in H_{0}^{1}(\Omega)$ with

$$
\begin{aligned}
& D T_{k}\left(v^{\varepsilon}\right)=\mathbb{1}_{\left\{v^{\varepsilon}<k\right\}}\left[\gamma\left(u^{\varepsilon}\right) b_{\varepsilon}\left(u^{\varepsilon}\right)+\left(1-b_{\varepsilon}\left(u^{\varepsilon}\right)\right) \beta(m-\varepsilon)\right] D T_{k / \alpha}\left(u^{\varepsilon}\right)^{+} \\
& D T_{k}\left(w^{\varepsilon}\right)=\mathbb{1}_{\left\{w^{\varepsilon}<k\right\}}\left[\beta\left(u^{\varepsilon}\right) b_{\varepsilon}\left(u^{\varepsilon}\right)+\left(1-b_{\varepsilon}\left(u^{\varepsilon}\right)\right) \beta(m-\varepsilon)\right] D T_{k / \alpha}\left(u^{\varepsilon}\right)^{+}
\end{aligned}
$$

a.e. in $\Omega$. In order to shorten the notation, we set for $\varepsilon>0$

$$
G^{\varepsilon}(r)=\int_{0}^{r^{+}}\left(\gamma(s) b_{\varepsilon}(s)+\left(1-b_{\varepsilon}(s)\right) \beta(m-\varepsilon)\right) \mathrm{d} s,
$$

which is the Lipschitz-continuous monotone function such that

$$
v^{\varepsilon}=G^{\varepsilon}\left(u^{\varepsilon}\right) .
$$

The proof is now divided into 3 steps.

Step 1. A priori estimates and pointwise convergence of $u^{\varepsilon}$.

All the estimates derived below are obtained through (1.18)-(1.21) with a classical technique (at least as far as a reader which is familiar with renormalized solutions is concerned). It consists in choosing $h=h_{p}$ in (1.21), then in plugging a test function which is bounded and with a gradient equal to a monotone function of $D T_{l}\left(u^{\varepsilon}\right)$ (as this is the case in (1.26), $(1.27)$ ) and then to pass to the limit in the obtained result making use of (1.20) (with $p$ in place of $n$ ) when $p$ tends to $+\infty$. It means that using formally such test functions directly in $(1.18)$ is a licit process. We refer to $[7,12,13]$ if necessary.

Choosing first $T_{k}\left(u^{\varepsilon}\right)$ in this process (i.e. $h=h_{p}$ with $T_{k}\left(u^{\varepsilon}\right)$ as a test function and letting $p \rightarrow+\infty$ for fixed $k$ and $\varepsilon$ ), we first obtain the classical estimate (see (1.4), (1.6))

$$
\begin{gathered}
\alpha \int_{\Omega}\left|D T_{k}\left(u^{\varepsilon}\right)\right|^{2} \mathrm{~d} x \leq k\|f\|_{L^{1}(\Omega)}, \\
\left(\mathbf{A}^{\varepsilon}\left(x, u^{\varepsilon}\right)\right)^{1 / 2} D T_{k}\left(u^{\varepsilon}\right) \text { is bounded in }\left(L^{2}(\Omega)\right)^{N},
\end{gathered}
$$

for any $k \geq 0$ and uniformly in $\varepsilon$.

From (1.30) we deduce with a classical argument (see e.g. [12]) that, for a subsequence still indexed by $\varepsilon$,

$$
\begin{aligned}
u^{\varepsilon} \longrightarrow u & \text { a.e. in } \Omega, \\
T_{k}\left(u^{\varepsilon}\right) \longrightarrow T_{k}(u) & \text { weakly in } H_{0}^{1}(\Omega),
\end{aligned}
$$

as $\varepsilon$ tends to 0 , where $u$ is a measurable function defined on $\Omega$ which is finite a.e. in $\Omega$ (and which belongs to $\left.W_{0}^{1, q}(\Omega), 1 \leq q<N /(N-1)\right)$.

To prove that $u$ is less or equal to $m$ is an easy task which is performed exactly as in [6]: when choosing $T_{2 m}^{+}\left(u^{\varepsilon}\right)-T_{m}^{+}\left(u^{\varepsilon}\right)$ instead of $T_{k}\left(u^{\varepsilon}\right)$ in the above process and by using the definition (1.16) of $\mathbf{A}^{\varepsilon}$, one obtain

$$
\beta(m-\varepsilon) \int_{\Omega}\left|D T_{2 m}^{+}\left(u^{\varepsilon}\right)-D T_{m}^{+}\left(u^{\varepsilon}\right)\right|^{2} \mathrm{~d} x \leq m\|f\|_{L^{1}(\Omega)} .
$$


Then in view of (1.32) and with the help of Poincaré's inequality together with the fact that $\beta(m-\varepsilon) \rightarrow+\infty$ as $\varepsilon$ tends to 0 , we deduce that

$$
T_{2 m}^{+}(u)-T_{m}^{+}(u)=0 \quad \text { a.e. in } \Omega,
$$

that is

$$
u \leq m \text { a.e. in } \Omega \text {. }
$$

Let us now take $T_{n}\left(w^{\varepsilon}-\left(u^{\varepsilon}\right)^{-}\right)$as a test function in (1.21) (with $h=h_{p}$ and then passing to the limit as $p$ goes infinity), we obtain

$$
\int_{\Omega} \mathbf{A}^{\varepsilon}\left(x, u^{\varepsilon}\right) D u^{\varepsilon} \cdot D T_{n}\left(w^{\varepsilon}-\left(u^{\varepsilon}\right)^{-}\right) \mathrm{d} x \leq n\|f\|_{L^{1}(\Omega)} .
$$

Since the support of $w^{\varepsilon}$ and $\left(u^{\varepsilon}\right)^{-}$are disjoint, we deduce that, using also (1.27)

$$
\begin{aligned}
\int_{\Omega} \mathbb{1}_{\left\{w^{\varepsilon}<n\right\}}\left[\beta\left(u^{\varepsilon}\right) b_{\varepsilon}\left(u^{\varepsilon}\right)\right. & +\left(1-b_{\varepsilon}\left(u^{\varepsilon}\right)\right] \mathbf{A}^{\varepsilon}\left(x, u^{\varepsilon}\right) D\left(u^{\varepsilon}\right)^{+} \cdot D T_{n / \alpha}\left(\left(u^{\varepsilon}\right)^{+}\right) \mathrm{d} x \\
& +\int_{\Omega} \mathbb{1}_{\left\{\left(u^{\varepsilon}\right)^{-}<n\right\}} \mathbf{A}^{\varepsilon}\left(x, u^{\varepsilon}\right) D\left(u^{\varepsilon}\right)^{-} \cdot D T_{n}\left(\left(u^{\varepsilon}\right)^{-}\right) \leq n\|f\|_{L^{1}(\Omega)} .
\end{aligned}
$$

Now the definition (1.16) of $\mathbf{A}^{\varepsilon}$ together with assumption (1.6) show that

$$
\beta(s) b_{\varepsilon}(s)|\xi|^{2}+\left(1-b_{\varepsilon}(s)\right) \beta(m-\varepsilon)|\xi|^{2} \leq \mathbf{A}^{\varepsilon}(x, s) \xi \cdot \xi,
$$

for any $s \in \mathbb{R}$; any $\xi \in \mathbb{R}^{N}$ and a.e. in $\Omega$.

Then (1.16), (1.27) and (1.35) yield

$$
\int_{\Omega}\left|D T_{n}\left(w^{\varepsilon}\right)\right|^{2} \mathrm{~d} x+\alpha \int_{\Omega}\left|D T_{n}\left(\left(u^{\varepsilon}\right)^{-}\right)\right|^{2} \mathrm{~d} x \leq n\|f\|_{L^{1}(\Omega)},
$$

which in turn implies (using again the fact that $w^{\varepsilon}$ and $\left(u^{\varepsilon}\right)^{-}$have disjoint supports)

$$
\min (1, \alpha) \int_{\Omega}\left|D T_{n}\left(w^{\varepsilon}-\left(u^{\varepsilon}\right)^{-}\right)\right|^{2} \mathrm{~d} x \leq n\|f\|_{L^{1}(\Omega)} .
$$

Poincaré's inequality and (1.37) lead to

$$
n^{2} \text { meas }\left\{x \in \Omega ;\left|w^{\varepsilon}-\left(u^{\varepsilon}\right)^{-}\right|>n\right\} \leq C n\|f\|_{L^{1}(\Omega)},
$$

where $C$ does not depend on $n$ and $\varepsilon$, and we obtain that

$$
\lim _{n \rightarrow+\infty} \sup _{\varepsilon}\left\{x \in \Omega ;\left|w^{\varepsilon}-\left(u^{\varepsilon}\right)^{-}\right|>n\right\}=0 .
$$

To obtain the analog of (1.38) with $v^{\varepsilon}$ in place of $w^{\varepsilon}$, we use the assumption $\int_{0}^{m} \gamma(s) \mathrm{d} s<$ $+\infty$. Indeed

$$
v^{\varepsilon}=w^{\varepsilon}+\int_{0}^{\left(u^{\varepsilon}\right)^{+}}(\gamma(s)-\beta(s)) b_{\varepsilon}(s) \mathrm{d} s \leq w^{\varepsilon}+\int_{0}^{m}(\gamma(s)-\beta(s)) \mathrm{d} s,
$$

where $\int_{0}^{m}(\gamma(s)-\beta(s)) \mathrm{d} s<+\infty$ by (1.5). It follows that (1.38) implies that

$$
\lim _{n \rightarrow+\infty} \sup _{\varepsilon}\left\{x \in \Omega ;\left|v^{\varepsilon}-\left(u^{\varepsilon}\right)^{-}\right|>n\right\}=0 .
$$

Another consequence of (1.36) is that (for a subsequence) $w^{\varepsilon}$ converges almost everywhere in $\Omega$ to a measurable function $w$ which is finite almost everywhere in $\Omega$. Then (1.39), in 
which $\left(u^{\varepsilon}\right)^{+}$can be replaced by $T_{m}\left(\left(u^{\varepsilon}\right)^{+}\right)$(because of the definition of $b_{\varepsilon}$ ), shows that (for a subsequence)

$$
v^{\varepsilon} \longrightarrow v \quad \text { a.e. in } \Omega \text {, }
$$

where $v=w+\int_{0}^{u^{+}}(\gamma(s)-\beta(s)) \mathrm{d} s$, because $T_{m}\left(\left(u^{\varepsilon}\right)^{+}\right) \rightarrow u^{+}$a.e. in $\Omega$ since $u \leq m$ a.e. in $\Omega$. Indeed $v$ is a measurable positive function which is finite almost everywhere in $\Omega$. Let us point out that the definitions (1.9) of $b_{\varepsilon}$ and (1.22) of $v^{\varepsilon}$, together with the convergences (1.32) and (1.41), show that

$$
\begin{aligned}
& v=\int_{0}^{u^{+}} \gamma(s) \mathrm{d} s \quad \text { a.e. in }\{x \in \Omega ; u(x)<m\}, \\
& w=\int_{0}^{u^{+}} \beta(s) \mathrm{d} s \quad \text { a.e. in }\{x \in \Omega ; u(x)<m\},
\end{aligned}
$$

but we do not know if this relations hold true on the subset $\{x \in \Omega ; u(x)=m\}$.

Now we choose $\theta_{n}\left(v^{\varepsilon}-\left(u^{\varepsilon}\right)^{-}\right)$as a test function in (1.21), it gives

$$
\frac{1}{n} \int_{\left\{n \leq\left|v^{\varepsilon}-\left(u^{\varepsilon}\right)^{-}\right| \leq 2 n\right\}} \mathbf{A}^{\varepsilon}\left(x, u^{\varepsilon}\right) D u^{\varepsilon} \cdot D\left(v^{\varepsilon}-\left(u^{\varepsilon}\right)^{-}\right) \mathrm{d} x \leq \int_{\left\{\left|v^{\varepsilon}-\left(u^{\varepsilon}\right)^{-}\right|>n\right\}}|f| \mathrm{d} x .
$$

Using now $f \in L^{1}(\Omega)$ and (1.40) we obtain

$$
\lim _{n \rightarrow+\infty} \sup _{\varepsilon} \frac{1}{n} \int_{\left\{n \leq\left|v^{\varepsilon}-\left(u^{\varepsilon}\right)^{-}\right| \leq 2 n\right\}} \mathbf{A}^{\varepsilon}\left(x, u^{\varepsilon}\right) D u^{\varepsilon} \cdot D\left(v^{\varepsilon}-\left(u^{\varepsilon}\right)^{-}\right) \mathrm{d} x=0 .
$$

Remark that the condition (1.43) is the analog of the standard one (i.e. for a matrix $\mathbf{A}(x, s)$ defined and continuous for $s \in \mathbb{R}$ ) upon replacing $\left(u^{\varepsilon}\right)^{+}$by $v^{\varepsilon}$.

To end this step we show below that the flux $\mathbf{A}^{\varepsilon}\left(x, u^{\varepsilon}\right) D u^{\varepsilon}$ is bounded in $\left(L^{2}(\Omega)\right)^{N}$ on the subsets where $v^{\varepsilon}-\left(u^{\varepsilon}\right)^{-}$is truncated. To this end we plug the test function $T_{k}\left(v^{\varepsilon}\right)$ in (1.21). We obtain using (1.26)

$$
\int_{\left\{\left|v^{\varepsilon}\right|<k\right\}} \mathbf{A}^{\varepsilon}\left(x, u^{\varepsilon}\right) D u^{\varepsilon} \cdot\left[b_{\varepsilon}\left(u^{\varepsilon}\right) \gamma\left(u^{\varepsilon}\right)+\left(1-b_{\varepsilon}\left(u^{\varepsilon}\right)\right) \beta(m-\varepsilon)\right] D\left(u^{\varepsilon}\right)^{+} \mathrm{d} x \leq k\|f\|_{L^{1}(\Omega)} .
$$

Now remark that (1.6) and the definition (1.16) of $\mathbf{A}^{\varepsilon}(x, s)$ leads to

$$
\mathbf{A}^{\varepsilon}(x, s) \xi \cdot \xi \leq\left[b_{\varepsilon}(s) \gamma(s)+\left(1-b_{\varepsilon}(s)\right) \beta(m-\varepsilon)\right]|\xi|^{2}
$$

for any $s \in \mathbb{R}$, any $\xi \in \mathbb{R}^{N}$ and a.e. in $\Omega$. Using (1.45) with $\xi=\left[\mathbf{A}^{\varepsilon}\left(x, u^{\varepsilon}\right)\right]^{1 / 2} D\left(u^{\varepsilon}\right)^{+}$in (1.44) yields

and then for any $k \geq 0$

$$
\int_{\Omega} \mathbb{1}_{\left\{v^{\varepsilon}<k\right\}}\left|\mathbf{A}^{\varepsilon}\left(x, u^{\varepsilon}\right) D\left(u^{\varepsilon}\right)^{+}\right|^{2} \mathrm{~d} x \leq k\|f\|_{L^{1}(\Omega)},
$$

$$
\mathbb{1}_{\left\{v^{\varepsilon}<k\right\}} \mathbf{A}^{\varepsilon}\left(x, u^{\varepsilon}\right) D\left(u^{\varepsilon}\right)^{+} \quad \text { is bounded in }\left(L^{2}(\Omega)\right)^{N}
$$

uniformly in $\varepsilon$. Now, since $\mathbb{1}_{\left\{\left|v^{\varepsilon}-\left(u^{\varepsilon}\right)^{-}\right|<k\right\}}=\mathbb{1}_{\left\{0 \leq v^{\varepsilon}<k\right\}}+\mathbb{1}_{\left\{-k<u^{\varepsilon}<0\right\}}$ a.e. in $\Omega$, the continuous character of $\mathbf{A}(x, s)$ for $s \in(-\infty, 0]$ and estimate (1.30) show that for any $k \geq 0$

$$
\mathbb{1}_{\left\{\left|v^{\varepsilon}-\left(u^{\varepsilon}\right)^{-}\right|<k\right\}} \mathbf{A}^{\varepsilon}\left(x, u^{\varepsilon}\right) D u^{\varepsilon} \quad \text { is bounded in }\left(L^{2}(\Omega)\right)^{N}
$$

uniformly in $\varepsilon$.

Step 2. Weak limit of the fields and proof of $\mathbb{1}_{\{-k<u<m\}} \mathbf{A}(x, u) D u \in\left(L^{2}(\Omega)\right)^{N}$. 
We first use the estimates (1.31) and (1.47) to extract another subsequence, still indexed by $\varepsilon$, such that $\left(\right.$ recall that $\left.\operatorname{supp}\left(h_{n}\right) \subset[-2 n, 2 n]\right)$,

$$
\left(\mathbf{A}^{\varepsilon}\left(x, u^{\varepsilon}\right)\right)^{1 / 2} D T_{k}\left(u^{\varepsilon}\right) \longrightarrow X_{k} \quad \text { weakly in }\left(L^{2}(\Omega)\right)^{N},
$$

and

$$
h_{n}\left(v^{\varepsilon}-\left(u^{\varepsilon}\right)^{-}\right) \mathbf{A}^{\varepsilon}\left(x, u^{\varepsilon}\right) D u^{\varepsilon} \longrightarrow \psi_{n} \quad \text { weakly in }\left(L^{2}(\Omega)\right)^{N},
$$

as $\varepsilon$ tends to 0 , where for any $k \geq 0$ and $n \geq 1, X_{k} \in\left(L^{2}(\Omega)\right)^{N}$ and $\psi_{n} \in\left(L^{2}(\Omega)\right)^{N}$.

Next we identify $\psi_{n}$ on the subset where $u<m$ and to this end we use the same technique as in [3]. Let $h$ be a $\mathcal{C}^{\infty}(\mathbb{R})$-function such that $\operatorname{supp}(h)$ is compact in $(-M, l)$ with $l<m$ and $M>0$. Then using the fact that $h(s) \mathbf{A}^{\varepsilon}(x, s)=h(s) \mathbf{A}\left(x, T_{l}\left(s^{+}\right)-T_{M}\left(s^{-}\right)\right)$for $\varepsilon$ small enough and the convergences (1.32), (1.33), (1.41), we have

$$
h\left(u^{\varepsilon}\right) h_{n}\left(v^{\varepsilon}-\left(u^{\varepsilon}\right)^{-}\right) \mathbf{A}^{\varepsilon}\left(x, u^{\varepsilon}\right) D u^{\varepsilon} \longrightarrow h(u) h_{n}\left(v-u^{-}\right) \mathbf{A}(x, u) D u \quad \text { weakly in }\left(L^{2}(\Omega)\right)^{N},
$$

as $\varepsilon$ tends to 0 and where $D u$ stands for $D T_{l}\left(u^{+}\right)-D T_{M}\left(u^{-}\right)$. It follows from (1.49) and (1.50) that

$$
\psi_{n}=h_{n}\left(v-u^{-}\right) \mathbf{A}(x, u) D u \quad \text { a.e. in }\{x \in \Omega ; u(x)<m\}
$$

since $l<m$ and $M$ are arbitrary. Let us point out that to obtain (1.50) and then (1.51), it is sufficient to know that $v^{\varepsilon}$ pointwise converges to $v$ one the subset $\{x \in \Omega ; u(x)<m\}$ (this will be used in the parabolic case).

Now, remark that on the subset $\{x \in \Omega ; u(x)<m\}$, we have $0 \leq v=\int_{0}^{u^{+}} \gamma(s) \mathrm{d} s<$ $\int_{0}^{m} \gamma(s) \mathrm{d} s$, and then for $n>\int_{0}^{m} \gamma(s) \mathrm{d} s, h_{n}\left(v-u^{-}\right)=h_{n}\left(-u^{-}\right)$on $\{x \in \Omega ; u(x)<m\}$. It follows that from (1.51)

$$
\psi_{n}=h_{n}\left(-u^{-}\right) \mathbf{A}(x, u) D u \quad \text { a.e. in }\{x \in \Omega ; u(x)<m\},
$$

which in turn implies that

$$
\mathbb{1}_{\{-k<u<m\}} \mathbf{A}(x, u) D u \in\left(L^{2}(\Omega)\right)^{N} .
$$

We now identify $X_{k}$. To this end, proceeding exactly as for $\psi_{n}$ above, we first have for any $k \geq 0$

$$
h_{n}\left(v^{\varepsilon}-\left(u^{\varepsilon}\right)^{-}\right) \mathbf{A}^{\varepsilon}\left(x, u^{\varepsilon}\right) D T_{k}\left(u^{\varepsilon}\right) \longrightarrow \psi_{n}^{k} \quad \text { weakly in }\left(L^{2}(\Omega)\right)^{N}
$$

as $\varepsilon$ tends to 0 with

$$
\psi_{n}^{k}=h_{n}\left(v-u^{-}\right) \mathbf{A}(x, u) D T_{k}(u) \quad \text { a.e. in }\{x \in \Omega ; u(x)<m\}
$$

Then, for $n>\max \left(k, \int_{0}^{m} \gamma(s) \mathrm{d} s\right)$ one has $h_{n}\left(v-u^{-}\right) D T_{k}(u)=D T_{k}(u)$ a.e. in $\{x \in$ $\Omega ; u(x)<m\}$. It follows that

$$
\psi_{n}^{k}=\mathbf{A}(x, u) D T_{k}(u) \quad \text { a.e. in }\{x \in \Omega ; u(x)<m\}
$$

for $n>\max \left(k, \int_{0}^{m} \gamma(s) \mathrm{d} s\right)$.

Secondly, we write

$$
h_{n}\left(v^{\varepsilon}-\left(u^{\varepsilon}\right)^{-}\right)\left(\mathbf{A}^{\varepsilon}\left(x, u^{\varepsilon}\right)\right)^{1 / 2} D T_{k}\left(u^{\varepsilon}\right)=h_{n}\left(v^{\varepsilon}-\left(u^{\varepsilon}\right)^{-}\right)\left(\mathbf{A}^{\varepsilon}\left(x, u^{\varepsilon}\right)\right)^{-1 / 2} \mathbf{A}^{\varepsilon}\left(x, u^{\varepsilon}\right) D T_{k}\left(u^{\varepsilon}\right)
$$


and we use the pointwise convergence of $u^{\varepsilon}$ to obtain $\left(\mathbf{A}^{\varepsilon}\left(x, u^{\varepsilon}\right)\right)^{-1 / 2} \rightarrow(\mathbf{A}(x, u))^{-1 / 2}$ a.e. in $\Omega$ (with indeed $(\mathbf{A}(x, u))^{-1 / 2}=0$ on the subset $\{x \in \Omega ; u(x)=m\}$ ). Passing to the limit in (1.57) as $\varepsilon$ tends to 0 (remark that $\left.\left|\left(\mathbf{A}^{\varepsilon}\left(x, u^{\varepsilon}\right)\right)^{-1 / 2} \xi\right|^{2} \leq|\xi|^{2} / \alpha\right)$ gives using (1.54) $(1.58)$ $h_{n}\left(v^{\varepsilon}-\left(u^{\varepsilon}\right)^{-}\right)\left(\mathbf{A}^{\varepsilon}\left(x, u^{\varepsilon}\right)\right)^{1 / 2} D T_{k}\left(u^{\varepsilon}\right) \longrightarrow(\mathbf{A}(x, u))^{-1 / 2} \psi_{n}^{k} \quad$ weakly in $\left(L^{2}(\Omega)\right)^{N}$ as $\varepsilon$ tends to 0 . Now for $n>\max \left(k, \int_{0}^{m} \gamma(s) \mathrm{d} s\right)$,

$$
(\mathbf{A}(x, u))^{-1 / 2} \psi_{n}^{k}=\mathbb{1}_{\{u<m\}}(\mathbf{A}(x, u))^{1 / 2} D T_{k}(u) \text { a.e. in } \Omega
$$

because of (1.56) in the the subset $\{x \in \Omega ; u(x)<m\}$ and the equality is trivial in $\{x \in$ $\Omega ; u(x)=m\}$ since both $(\mathbf{A}(x, u))^{-1 / 2}$ and $D T_{k}(u)$ are equal to 0 . In view of (1.48) and (1.58) we deduce that

$$
X_{k}=\mathbb{1}_{\{u<m\}}(\mathbf{A}(x, u))^{1 / 2} D T_{k}(u) \quad \text { a.e. in } \Omega .
$$

Step 3. End of the proof.

We choose $h(r)=h_{n}\left(G^{\varepsilon}(r)-r^{-}\right)$in $(1.21)$ (recall that this is licit because $h \in W^{1, \infty}(\mathbb{R})$ and $\operatorname{supp}(h) \subset[-2 n, 2 n / \alpha])$. Then let $z$ be an element of $L^{\infty}(\Omega) \cap H_{0}^{1}(\Omega)$. Plugging $z$ as a test function in (1.21) with $h$ defined above, we obtain using (1.29)

$$
\begin{aligned}
& \int_{\Omega} h_{n}\left(v^{\varepsilon}-\left(u^{\varepsilon}\right)^{-}\right) \mathbf{A}^{\varepsilon}\left(x, u^{\varepsilon}\right) D u^{\varepsilon} \cdot D z \mathrm{~d} x \\
&=\int_{\Omega} f h_{n}\left(v^{\varepsilon}-\left(u^{\varepsilon}\right)^{-}\right) z \mathrm{~d} x+\int_{\Omega} z \mathbf{A}^{\varepsilon}\left(x, u^{\varepsilon}\right) D u^{\varepsilon} \cdot D h_{n}\left(v^{\varepsilon}-\left(u^{\varepsilon}\right)^{-}\right) \mathrm{d} x .
\end{aligned}
$$

First recall that the convergences (1.32) and (1.41) give

$$
\int_{\Omega} f h_{n}\left(v^{\varepsilon}-\left(u^{\varepsilon}\right)^{-}\right) z \mathrm{~d} x \longrightarrow \int_{\Omega} f h_{n}\left(v-u^{-}\right) z \mathrm{~d} x
$$

as $\varepsilon$ tends to 0 .

Then, setting

$$
\omega(n)=\sup _{\varepsilon} \frac{1}{n} \int_{\left\{n<\left|v^{\varepsilon}-\left(u^{\varepsilon}\right)^{-}\right|<2 n\right\}} \mathbf{A}^{\varepsilon}\left(x, u^{\varepsilon}\right) D u^{\varepsilon} \cdot D\left(v^{\varepsilon}-\left(u^{\varepsilon}\right)^{-}\right) \mathrm{d} x,
$$

we deduce that from (1.49), upon extracting another subsequence of $\varepsilon$, still denoted by $\varepsilon$

$$
\begin{aligned}
-\omega(n)\|z\|_{L^{\infty}(\Omega)} \leq \lim _{\varepsilon \rightarrow 0} \int_{\Omega} h_{n}\left(v^{\varepsilon}-\left(u^{\varepsilon}\right)^{-}\right) \mathbf{A}^{\varepsilon}(x, & \left.u^{\varepsilon}\right) D u^{\varepsilon} D z \mathrm{~d} x \\
& -\int_{\Omega} f h_{n}\left(v-u^{-}\right) z \mathrm{~d} x \leq \omega(n)\|z\|_{L^{\infty}(\Omega)} .
\end{aligned}
$$

Now $h_{n}\left(v-u^{-}\right) \rightarrow 1$ a.e. in $\Omega$ as $n$ tends to $+\infty$ (recall that both $u$ and $v$ are finite a.e. in $\Omega$ ) while $\omega(n) \rightarrow 0$ as $n$ tends to $+\infty$ (see (1.43)), it follows that

$$
\lim _{n \rightarrow+\infty} \lim _{\varepsilon \rightarrow 0} \int_{\Omega} h_{n}\left(v^{\varepsilon}-\left(u^{\varepsilon}\right)^{-}\right) \mathbf{A}^{\varepsilon}\left(x, u^{\varepsilon}\right) D u^{\varepsilon} D z \mathrm{~d} x=\int_{\Omega} f z \mathrm{~d} x .
$$

From (1.61), we will deduce that $u$ satisfies (1.13)-(1.15) and the strong convergence of the energy. 
Firstly choose $z=h(u) \varphi$ where $h \in W^{1, \infty}(\mathbb{R})$ has a compact support and satisfies $h(m)=$ 0 and $\varphi \in \mathcal{C}_{0}^{\infty}(\Omega)$. By (1.33) we have $h(u) \varphi \in H_{0}^{1}(\Omega) \cap L^{\infty}(\Omega)$. Then, (1.49) and (1.61) lead to

$$
\lim _{n \rightarrow+\infty} \int_{\Omega} \psi_{n} D(h(u) \varphi) \mathrm{d} x=\int_{\Omega} f h(u) \varphi \mathrm{d} x .
$$

Using now the identification (1.52) of $\psi_{n}$, it follows that, for $k \geq 0$ such that $\operatorname{supp}(h) \subset$ $[-k, k]$ and $n \geq k$

$$
\begin{aligned}
\psi_{n} D[h(u) \varphi] & =\psi_{n} h(u) D \varphi+\psi_{n} \varphi D h\left(T_{k}(u)\right) \\
& =\mathbb{1}_{\{u<m\}} \mathbf{A}(x, u) D[h(u) \varphi] \quad \text { a.e. in } \Omega
\end{aligned}
$$

because $h(m)=0$ and $D h\left(T_{k}(u)\right)=0$ a.e. on the subset $\{x \in \Omega ; u(x)=m\}$ (since $\left.T_{k}(u) \in H_{0}^{1}(\Omega)\right)$.

From (1.62) and (1.63), we obtain that

$$
\int_{\Omega} \mathbb{1}_{\{u<m\}} \mathbf{A}(x, u) D u \cdot D[h(u) \varphi] \mathrm{d} x=\int_{\Omega} f h(u) \varphi \mathrm{d} x
$$

for any $\varphi \in \mathcal{C}_{0}^{\infty}(\Omega)$. This shows that $(1.15)$ is satisfied in $\mathcal{D}^{\prime}(\Omega)$

Secondly, we choose $z=\theta_{p}\left(-u^{-}\right)$(see (1.7)) for a fixed integer $p \geq 1$ and we obtain

$$
\lim _{n \rightarrow+\infty} \int_{\Omega} \psi_{n} D \theta_{p}\left(-u^{-}\right) \mathrm{d} x=\int_{\Omega} f \theta_{p}\left(-u^{-}\right) \mathrm{d} x .
$$

Using the identification of $\psi_{n}$ as above, it gives

$$
\int_{\Omega} \mathbf{A}(x, u) D u \cdot D \theta_{p}\left(-u^{-}\right) \mathrm{d} x=\int_{\Omega} f \theta_{p}\left(-u^{-}\right) \mathrm{d} x
$$

and, then letting $p$ tends to $+\infty$

$$
\lim _{p \rightarrow+\infty} \frac{1}{p} \int_{\{-2 p<u<-p\}} \mathbf{A}(x, u) D u \cdot D u \mathrm{~d} x=0,
$$

since again $u$ is finite a.e. in $\Omega$, and (1.13) is established.

Thirdly to obtain (1.14), we take $z_{p}=\left(1-b_{1 / p}\left(u^{+}\right)\right) \varphi$ where $p$ is a fixed integer $\geq 1$ (see (1.9)) and $\varphi \in H^{1}(\Omega) \cap L^{\infty}(\Omega)$ is such that $D \varphi=0$ a.e. in $\{x \in \Omega ; u(x)=m\}$. Indeed $\left\|z_{p}\right\|_{L^{\infty}(\Omega)} \leq\|\varphi\|_{L^{\infty}(\Omega)}$ and

$$
D z_{p}=p \mathbb{1}_{\{m-2 / p<u<m-1 / p\}} D u \varphi+\left(1-b_{1 / p}\left(u^{+}\right)\right) D \varphi
$$

so that $D z_{p}=0$ a.e. on $\{x \in \Omega ; u(x)=m\}$. It follows that from (1.61)

$$
\begin{aligned}
& p \int_{\Omega} \mathbb{1}_{\{m-2 / p<u<m-1 / p\}} \mathbf{A}(x, u) D u \cdot D u \varphi \mathrm{d} x \\
& +\int_{\Omega}\left(1-b_{1 / p}\left(u^{+}\right)\right) \mathbb{1}_{\{u<m\}} \mathbf{A}(x, u) D u \cdot D \varphi \mathrm{d} x \\
& \quad=\int_{\Omega} f\left(1-b_{1 / p}\left(u^{+}\right)\right) \varphi \mathrm{d} x .
\end{aligned}
$$

Now, as $p$ tends to $+\infty,\left(1-b_{1 / p}\left(u^{+}\right)\right) \rightarrow \mathbb{1}_{\{u=m\}}$ a.e. in $\Omega$ and (1.65) gives

$$
\lim _{p \rightarrow+\infty} p \int_{\Omega} \mathbb{1}_{\{m-2 / p<u<m-1 / p\}} \mathbf{A}(x, u) D u \cdot D u \varphi \mathrm{d} x=\int_{\Omega} f \mathbb{1}_{\{u=m\}} \varphi \mathrm{d} x
$$

which is (1.14). 
Finally, in order to prove the strong convergence of the energy we choose $z=T_{k}(u)$. The relation (1.61) gives as above (remark that $D T_{k}(u)=0$ a.e. in $\{x \in \Omega ; u(x)=m\}$ )

$$
\lim _{n \rightarrow+\infty} \int_{\Omega} \psi_{n} D T_{k}(u) \mathrm{d} x=\int_{\Omega} f T_{k}(u) \mathrm{d} x .
$$

The identification (1.52) of $\psi_{n}$ then leads to

$$
\lim _{n \rightarrow+\infty} \int_{\Omega} h_{n}\left(-u^{-}\right) \mathbb{1}_{\{u<m\}} \mathbf{A}(x, u) D u \cdot D T_{k}(u) \mathrm{d} x=\int_{\Omega} f T_{k}(u) \mathrm{d} x
$$

and then for $n>k$

$$
\int_{\Omega} \mathbb{1}_{\{u<m\}} \mathbf{A}(x, u) D T_{k}(u) \cdot D T_{k}(u) \mathrm{d} x=\int_{\Omega} f T_{k}(u) \mathrm{d} x
$$

for any $k \geq 0$.

Indeed, recalling the process that leads to (1.30) and passing to the limit first as $p \rightarrow+\infty$ and then as $\varepsilon$ tends to 0 , using the pointwise convergence of $u^{\varepsilon}$, permits to obtain the classical convergence

$$
\lim _{\varepsilon \rightarrow 0} \int_{\Omega} \mathbf{A}^{\varepsilon}\left(x, u^{\varepsilon}\right) D T_{k}\left(u^{\varepsilon}\right) \cdot D T_{k}\left(u^{\varepsilon}\right) \mathrm{d} x=\int_{\Omega} f T_{k}(u) \mathrm{d} x .
$$

From the identification (1.59) of $X_{k}$ we deduce from (1.66) and (1.67) that for any $k \geq 0$

$$
\left(\mathbf{A}^{\varepsilon}\left(x, u^{\varepsilon}\right)\right)^{1 / 2} D T_{k}\left(u^{\varepsilon}\right) \longrightarrow \mathbb{1}_{\{u<m\}}(\mathbf{A}(x, u))^{1 / 2} D T_{k}(u) \text { strongly in }\left(L^{2}(\Omega)\right)^{N},
$$

as $\varepsilon$ tends to 0 .

Remark that (1.68) implies that for any $k \geq 0$

$$
T_{k}\left(u^{\varepsilon}\right) \longrightarrow T_{k}(u) \text { strongly in } H_{0}^{1}(\Omega) \text {, as } \varepsilon \text { tends to } 0 .
$$

The proof of Theorem 1.4 is achieved.

\section{Part 2. The parabolic case}

\subsection{Assumption on the Data AND Definition of A SOlution}

The second part of the paper is devoted to investigate a parabolic version of (1.1) namely the problem

$$
\begin{cases}\frac{\partial u}{\partial t}-\operatorname{div}[\mathbf{A}(t, x, u) D u]=f & \text { in }(0, T) \times \Omega, \\ u=0 & \text { on }(0, T) \times \partial \Omega \\ u(t=0)=u_{0} & \text { in } \Omega,\end{cases}
$$

where now $T>0$ and

$$
\begin{gathered}
f \in L^{1}((0, T) \times \Omega) ; \\
u_{0} \in L^{1}(\Omega) \text { and } u_{0} \leq m \text { a.e. in } \Omega ;
\end{gathered}
$$

$\mathbf{A}:(t, x, s) \rightarrow \mathbf{A}(t, x, s)$ is a Carathéodory function from $(0, T) \times \Omega \times$ $(-\infty, m)$ into $\mathbb{R}_{S}^{N \times N}$, such that there exists two positive functions $\beta$ and $\gamma$ in $\mathcal{C}^{0}((-\infty, m))$ which satisfy $(1.4),(1.5)$ and

We denote by $Q$ the set $(0, T) \times \Omega$. We use the following definition of a solution of $(2.1)$. 
Definition 2.1. A function $u$ in $L^{\infty}\left(0, T ; L^{1}(\Omega)\right)$ is a renormalized solution of (2.1) if

$$
\begin{gathered}
\forall k \geq 0, \quad T_{k}(u) \in L^{2}\left(0, T ; H_{0}^{1}(\Omega)\right) ; \\
u \leq m \quad \text { a.e. in } \quad Q ; \\
\forall k \geq 0, \quad \mathbb{1}_{\{-k<u<m\}} \mathbf{A}(t, x, u) D u \in\left(L^{2}(Q)\right)^{N} \\
\lim _{n \rightarrow+\infty} \frac{1}{n} \int_{\{-2 n<u<-n\}} \mathbf{A}(t, x, u) D u \cdot D u \mathrm{~d} x \mathrm{~d} t=0 ;
\end{gathered}
$$

for any $\varphi \in \mathcal{C}_{0}^{\infty}([0, T))$

$$
\lim _{n \rightarrow+\infty} n \int_{\{m-2 / n<u<m-1 / n\}} \varphi \mathbf{A}(t, x, u) D u \cdot D u \mathrm{~d} x \mathrm{~d} t=\int_{\{u=m\}} f \varphi \mathrm{d} x \mathrm{~d} t .
$$

$\forall S \in W^{2, \infty}(\mathbb{R})$ such that $\operatorname{supp}\left(S^{\prime}\right)$ is compact and $S^{\prime}(m)=0, \forall \varphi \in W^{1, \infty}(Q)$ such that $\varphi(T)=0$ and $S^{\prime}(0) \varphi=0$ on $\partial \Omega, u$ satisfies

$$
\begin{aligned}
-\int_{Q} \varphi_{t} S(u) \mathrm{d} x \mathrm{~d} t-\int_{\Omega} \varphi(0) & S\left(u_{0}\right) \mathrm{d} x \\
& \quad+\int_{Q} \mathbf{A}(t, x, u) D u \cdot D\left[S^{\prime}(u) \varphi\right] \mathrm{d} x \mathrm{~d} t=\int_{Q} f S^{\prime}(u) \varphi \mathrm{d} x \mathrm{~d} t
\end{aligned}
$$

Remark 2.2 (Comments on Definition 2.1). Conditions (2.6) and (2.9) are classical in the framework of renormalized solutions. Indeed (2.7) and (2.10) is the analog of (1.11) and (1.14) in the elliptic case, and due to (2.2), (2.4) and (2.6) every term in (2.11) makes sense. Let us point out that the main difference between Definition 1.2 and 2.1 is that $(2.10)$ is analog to (1.14) but with $\varphi \in \mathcal{C}_{0}^{\infty}([0, T))$ which does not depend on the variable $x$. Actually we are not able to prove (2.10) with any function $\varphi \in L^{2}\left(0, T ; H^{1}(\Omega)\right) \cap L^{\infty}(Q)$ such that $D \varphi=0$ a.e. in $\{(t, x) ; u(t, x)=m\}$ because of a lack of regularity on $u$ with respect to $t$ in the parabolic case. Then Definition 2.1 is less precise that Definition 1.2.

Moreover remark that, since we are dealing with homogeneous Dirichlet condition $(u=0$ on $(0, T) \times \partial \Omega)$, the condition $S^{\prime}(u) \varphi \in L^{2}\left(0, T ; H_{0}^{1}(\Omega)\right)$ just rewrites as $S^{\prime}(0) \varphi=0$ on $(0, T) \times \partial \Omega$ (indeed $S^{\prime}(u) \varphi \in L^{2}\left(0, T ; H^{1}(\Omega)\right)$ by $\left.(2.6)\right)$.

\subsection{Existence RESUlt}

We prove the following result

Theorem 2.3. Under the assumptions (2.2)-(2.5), there exists at least a solution of (2.1) in the sense of Definition 2.1.

Proof of Theorem 2.3. We proceed through the same approximation process as in the proof of Theorem 1.4 and define $\mathbf{A}^{\varepsilon}(t, x, s)$ by the expression given in (1.16) (with $\mathbf{A}(t, x, s)$ in place of $\mathbf{A}(x, s))$. Due to (1.17), the approximate problem

$$
\begin{cases}\frac{\partial u^{\varepsilon}}{\partial t}-\operatorname{div}\left[\mathbf{A}^{\varepsilon}\left(t, x, u^{\varepsilon}\right) D u^{\varepsilon}\right]=f & \text { in } Q, \\ u^{\varepsilon}=0 & \text { on }(0, T) \times \partial \Omega, \\ u^{\varepsilon}(t=0)=u_{0} & \text { in } \Omega,\end{cases}
$$


admits at least a renormalized solution (see e.g. $[4,5]$ ). Recall that such a solution satisfies

$$
\begin{gathered}
u^{\varepsilon} \in L^{\infty}\left(0, T ; L^{1}(\Omega)\right) \\
\forall k \geq 0 ; \quad T_{k}\left(u^{\varepsilon}\right) \in L^{2}\left(0, T ; H_{0}^{1}(\Omega)\right) \\
\lim _{n \rightarrow+\infty} \frac{1}{n} \int_{\left\{n<\left|u^{\varepsilon}\right|<2 n\right\}} \mathbf{A}^{\varepsilon}\left(t, x, u^{\varepsilon}\right) D u^{\varepsilon} \cdot D u^{\varepsilon} \mathrm{d} x \mathrm{~d} t=0 ;
\end{gathered}
$$

$\forall S \in W^{2, \infty}(\mathbb{R})$ such that $\operatorname{supp}\left(S^{\prime}\right)$ is compact, $\forall \varphi \in W^{1, \infty}(Q)$ such that $\varphi(T)=0$ and $S^{\prime}\left(u^{\varepsilon}\right) \varphi \in L^{2}\left(0, T ; H_{0}^{1}(\Omega)\right), u^{\varepsilon}$ satisfies

$$
\begin{aligned}
-\int_{Q} \varphi_{t} S\left(u^{\varepsilon}\right) \mathrm{d} x \mathrm{~d} t & -\int_{\Omega} \varphi(0) S\left(u_{0}\right) \mathrm{d} x \\
& +\int_{Q} \mathbf{A}^{\varepsilon}\left(t, x, u^{\varepsilon}\right) D u^{\varepsilon} \cdot D\left[S^{\prime}\left(u^{\varepsilon}\right) \varphi\right] \mathrm{d} x \mathrm{~d} t=\int_{Q} f S^{\prime}\left(u^{\varepsilon}\right) \varphi \mathrm{d} x \mathrm{~d} t
\end{aligned}
$$

In order to prove that, for a subsequence still indexed by $\varepsilon$, the sequence $u^{\varepsilon}$ converges to a solution in the sense of Definition 2.1 we proceed in 5 Steps.

Step 1. A priori estimates

Let us define the two sequences $v^{\varepsilon}$ and $w^{\varepsilon}$ through the formulae (1.22) and (1.23) (now $v^{\varepsilon}$ and $w^{\varepsilon}$ are defined on $Q$ ). Since (1.24) and (1.25) still hold true (see assumptions (2.4) and (2.5)), (2.6) implies that $T_{k}\left(v^{\varepsilon}\right) \in L^{2}\left(0, T ; H_{0}^{1}(\Omega)\right), T_{k}\left(w^{\varepsilon}\right) \in L^{2}\left(0, T ; H_{0}^{1}(\Omega)\right)$ and

$$
\begin{gathered}
D T_{k}\left(v^{\varepsilon}\right)=\mathbb{1}_{\left\{v^{\varepsilon}<k\right\}}\left[\gamma\left(u^{\varepsilon}\right) b_{\varepsilon}\left(u^{\varepsilon}\right)+\left(1-b_{\varepsilon}\left(u^{\varepsilon}\right)\right) \beta(m-\varepsilon)\right] D T_{k / \alpha}\left(u^{\varepsilon}\right)^{+} \\
D T_{k}\left(w^{\varepsilon}\right)=\mathbb{1}_{\left\{w^{\varepsilon}<k\right\}}\left[\beta\left(u^{\varepsilon}\right) b_{\varepsilon}\left(u^{\varepsilon}\right)+\left(1-b_{\varepsilon}\left(u^{\varepsilon}\right)\right) \beta(m-\varepsilon)\right] D T_{k / \alpha}\left(u^{\varepsilon}\right)^{+}
\end{gathered}
$$

a.e. in $Q$.

The techniques used to derive all the estimates contained in this section is similar to the one used in the elliptic case. It consists in choosing $S^{\prime}(r)=h_{p}^{\prime}(r) \mathcal{Z}(r)$ in $(2.16)$, where $h_{p}$ is defined in (1.7) and where $\mathcal{Z}$ is a monotone bounded and Lipschitz continuous function defined on $\mathbb{R}$. The test function $\varphi$ is always equal to $\varphi=\min \left(\frac{(T-\delta-t)^{+}}{\delta}, 1\right)$ (which is then independent of $x$ ). Compared to the elliptic case, this means that the choice of the non linear test function of $u^{\varepsilon}$ is included in the function $S$ and this is the advantage of the formulation (2.16) : it has already used an integration by part (in time) formula (see Section 2 of [5]). In this process, the parameter $\delta$ first tends to 0 . Then we let $p$ tends first to $+\infty$ using (2.15), for a fixed $\varepsilon$ and a fixed function $\mathcal{Z}$.

We begin with classical estimates by choosing $S^{\prime}(r)=h_{p}(r) T_{k}(r)$ in $(2.16)$ (that is $\mathcal{Z}(r)=$ $\left.T_{k}(r)\right)$. We obtain

$$
\begin{aligned}
\frac{1}{\delta} \int_{T-2 \delta}^{T-\delta} \int_{\Omega} & \left(\int_{0}^{u^{\varepsilon}} h_{p}(r) T_{k}(r) \mathrm{d} r\right) \mathrm{d} x \mathrm{~d} t \\
& +\int_{0}^{T-2 \delta} \int_{\Omega} \mathbf{A}^{\varepsilon}\left(t, x, u^{\varepsilon}\right) D u^{\varepsilon} \cdot D\left[h_{p}\left(u^{\varepsilon}\right) T_{k}\left(u^{\varepsilon}\right)\right] \mathrm{d} x \mathrm{~d} t \\
& \leq k\left[\|f\|_{L^{1}(Q)}+\left\|u_{0}\right\|_{L^{1}(\Omega)}\right]
\end{aligned}
$$


Letting $\delta$ tend to 0 , it gives as soon as $p>k$

$$
\begin{aligned}
& \int_{0}^{T} \int_{\Omega} \mathbf{A}^{\varepsilon}\left(t, x, u^{\varepsilon}\right) D T_{k}\left(u^{\varepsilon}\right) \cdot D T_{k}\left(u^{\varepsilon}\right) \mathrm{d} x \mathrm{~d} t \\
& \leq k\left[\|f\|_{L^{1}(Q)}+\left\|u_{0}\right\|_{L^{1}(\Omega)}\right]+\frac{k}{p} \int_{\left\{(t, x) ; p<\left|u^{\varepsilon}\right|<2 p\right\}} \mathbf{A}^{\varepsilon}\left(t, x, u^{\varepsilon}\right) D u^{\varepsilon} \cdot D u^{\varepsilon} \mathrm{d} x \mathrm{~d} t .
\end{aligned}
$$

Now for fixed $\varepsilon$ and $k$ we let $p$ tends to $+\infty$ and it yields using (2.15)

$$
\int_{0}^{T} \int_{\Omega} \mathbf{A}^{\varepsilon}\left(t, x, u^{\varepsilon}\right) D T_{k}\left(u^{\varepsilon}\right) \cdot D T_{k}\left(u^{\varepsilon}\right) \mathrm{d} x \mathrm{~d} t \leq k\left[\|f\|_{L^{1}(Q)}+\left\|u_{0}\right\|_{L^{1}(\Omega)}\right] .
$$

In view of (1.16) (which is now also uniform in $t$ ), we deduce from (2.20) that

$$
\alpha \int_{Q}\left|D T_{k}\left(u^{\varepsilon}\right)\right|^{2} \mathrm{~d} x \mathrm{~d} t \leq k\left[\|f\|_{L^{1}(Q)}+\left\|u_{0}\right\|_{L^{1}(\Omega)}\right] .
$$

Remark that, replacing $T$ by $0<T^{\prime}<T$ in the function $\varphi$, the inequality (2.19) also leads to the classical estimate

$$
\left\|u^{\varepsilon}\right\|_{L^{\infty}\left(0, T ; L^{1}(\Omega)\right)} \leq\|f\|_{L^{1}(Q)}+\left\|u_{0}\right\|_{L^{1}(\Omega)} .
$$

Now we choose $S^{\prime}(r)=h_{p}(r)\left(T_{2 m}^{+}(r)-T_{m}^{+}(r)\right)$ in (2.16) and we proceed as above. After letting $\delta$ and $p$ tend to 0 and $+\infty$ respectively, we obtain

$$
\int_{Q} \mathbf{A}^{\varepsilon}\left(t, x, u^{\varepsilon}\right) D u^{\varepsilon} \cdot D\left[T_{2 m}^{+}\left(u^{\varepsilon}\right)-T_{m}^{+}\left(u^{\varepsilon}\right)\right] \mathrm{d} x \mathrm{~d} t \leq m\left[\|f\|_{L^{1}(Q)}+\left\|u_{0}\right\|_{L^{1}(\Omega)}\right] .
$$

Then, using the definition of $\mathbf{A}^{\varepsilon}$ exactly as in the elliptic case, we deduce that

$$
\beta(m-\varepsilon) \int_{Q}\left|T_{2 m}^{+}\left(u^{\varepsilon}\right)-T_{m}\left(u^{\varepsilon}\right)\right|^{2} \mathrm{~d} x \mathrm{~d} t \leq m\left[\|f\|_{L^{1}(Q)}+\left\|u_{0}\right\|_{L^{1}(\Omega)}\right] .
$$

Let us now define the Lipschitz-continuous monotone function $H^{\varepsilon}$ by

$$
H^{\varepsilon}(r)=\int_{0}^{r^{+}}\left(\beta(s) b_{\varepsilon}(s)+\left(1-b_{\varepsilon}(s)\right) \beta(m-\varepsilon)\right) \mathrm{d} s,
$$

so that

$$
w^{\varepsilon}=H^{\varepsilon}\left(u^{\varepsilon}\right) \text { a.e. in } Q .
$$

We choose $S^{\prime}(r)=h_{p}(r) T_{n}\left(H^{\varepsilon}(r)-r^{-}\right)$in (2.16) and we obtain as above, and using (2.25)

$$
\int_{Q} \mathbf{A}^{\varepsilon}\left(t, x, u^{\varepsilon}\right) D u^{\varepsilon} \cdot D T_{n}\left(w^{\varepsilon}-\left(u^{\varepsilon}\right)^{-}\right) \mathrm{d} x \mathrm{~d} t \leq n\left[\|f\|_{L^{1}(Q)}+\left\|u_{0}\right\|_{L^{1}(\Omega)}\right] .
$$

In (2.26) we have used the fact that $T_{n}\left(H^{\varepsilon}(r)-r^{-}\right)$has a derivative with compact support (see (1.24)). Proceeding now exactly as in the elliptic case (see (1.35), (1.37) and (1.38)), we deduce that from $(2.26)$

$$
\lim _{n \rightarrow+\infty} \sup _{\varepsilon}\left\{(t, x) \in Q ;\left|w^{\varepsilon}-\left(u^{\varepsilon}\right)^{-}\right|>n\right\}=0
$$

and then

$$
\lim _{n \rightarrow+\infty} \sup _{\varepsilon}\left\{(t, x) \in Q ;\left|v^{\varepsilon}-\left(u^{\varepsilon}\right)^{-}\right|>n\right\}=0 .
$$


This last result permits to obtain the energy condition when $v^{\varepsilon}-\left(u^{\varepsilon}\right)^{-}$is "large" through setting $S^{\prime}(r)=h_{p}(r) \theta_{n}\left(G^{\varepsilon}(r)-r^{-}\right)$in (2.16) which indeed gives (see the definition of $G^{\varepsilon}$ in $(1.28)$ and $(1.29))$

$$
\begin{aligned}
& \frac{1}{n} \int_{\left\{(t, x) ; n<\left|v^{\varepsilon}-\left(u^{\varepsilon}\right)^{-}\right|<2 n\right\}} \mathbf{A}^{\varepsilon}\left(t, x, u^{\varepsilon}\right) D u^{\varepsilon} \cdot D\left(v^{\varepsilon}-\left(u^{\varepsilon}\right)^{-}\right) \mathrm{d} x \mathrm{~d} t \\
& \quad \leq \int_{\left\{(t, x) ; n<\left|v^{\varepsilon}-\left(u^{\varepsilon}\right)^{-}\right|\right\}}|f| \mathrm{d} x \mathrm{~d} t+\int_{\Omega} \int_{0}^{\left|u_{0}\right|}\left|\theta_{n}\left(G^{\varepsilon}(r)-r^{-}\right)\right| \mathrm{d} r \mathrm{~d} x .
\end{aligned}
$$

As far as the first term of the right hand side of (2.29) is concerned, we use $f \in L^{1}(Q)$ and (2.28) to obtain

$$
\lim _{n \rightarrow+\infty} \sup _{\varepsilon} \int_{\left\{(t, x) ; n<\left|v^{\varepsilon}-\left(u^{\varepsilon}\right)^{-}\right|\right\}}|f| \mathrm{d} x \mathrm{~d} t=0 .
$$

For the second term, we recall that the support of $G^{\varepsilon}(r)$ and of $r^{-}$are disjoints so that

$$
\int_{\Omega} \int_{0}^{\left|u_{0}\right|}\left|\theta_{n}\left(G^{\varepsilon}(r)-r^{-}\right)\right| \mathrm{d} r \mathrm{~d} x \leq \int_{\Omega} \int_{0}^{\left|u_{0}\right|} \theta_{n}\left(G^{\varepsilon}(r)\right) \mathrm{d} r \mathrm{~d} x+\int_{\left\{u_{0}<-n\right\}}\left|u_{0}\right| \mathrm{d} x .
$$

Since $u_{0} \leq m$ almost everywhere in $\Omega$ and $\int_{0}^{+\infty} \gamma(s) \mathrm{d} s<+\infty$ we first have

$$
\sup _{\varepsilon} \int_{\Omega} \int_{0}^{\left|u_{0}\right|} \theta_{n}\left(G^{\varepsilon}(r)\right) \mathrm{d} r \mathrm{~d} x=0
$$

for $n>\int_{0}^{+\infty} \gamma(s) \mathrm{d} s$, while

$$
\lim _{n \rightarrow+\infty} \int_{\left\{u_{0}<-n\right\}}\left|u_{0}\right| \mathrm{d} x=0
$$

because $u_{0} \in L^{1}(\Omega)$.

In view of $(2.29),(2.30)$ and $(2.31)$, we conclude that

$$
\lim _{n \rightarrow+\infty} \sup _{\varepsilon} \frac{1}{n} \int_{\left\{(t, x) ; n<\left|v^{\varepsilon}-\left(u^{\varepsilon}\right)^{-}\right|<2 n\right\}} \mathbf{A}^{\varepsilon}\left(t, x, u^{\varepsilon}\right) D u^{\varepsilon} \cdot D\left(v^{\varepsilon}-\left(u^{\varepsilon}\right)^{-}\right) \mathrm{d} x \mathrm{~d} t=0 .
$$

Remark that repeating the above argument with $S^{\prime}(r)=h_{p}(r) \theta_{n}(r)$ leads to

$$
\frac{1}{n} \int_{\left\{n<\left|u^{\varepsilon}\right|<2 n\right\}} \mathbf{A}^{\varepsilon}\left(t, x, u^{\varepsilon}\right) D u^{\varepsilon} \cdot D u^{\varepsilon} \mathrm{d} x \mathrm{~d} t \leq \int_{Q} f \theta_{n}\left(u^{\varepsilon}\right) \mathrm{d} x \mathrm{~d} t+\int_{\left\{\left|u_{0}\right|>n\right\}}\left|u_{0}\right| \mathrm{d} x .
$$

To end this subsection, we derive the analog of (1.47) of the elliptic case. To this end, we take $S^{\prime}(r)=h_{p}(r) T_{k}\left(G^{\varepsilon}(r)\right)$ in $(2.16)$ and this yields

$$
\int_{Q} \mathbf{A}^{\varepsilon}\left(t, x, u^{\varepsilon}\right) D u^{\varepsilon} \cdot D T_{k}\left(v^{\varepsilon}\right) \mathrm{d} x \mathrm{~d} t \leq k\left[\|f\|_{L^{1}(Q)}+\left\|u_{0}\right\|_{L^{1}(\Omega)}\right] .
$$

Reproducing the arguments used in (1.44), (1.45), (1.46) of the elliptic case gives for any $k \geq 0$

$$
\mathbb{1}_{\left\{\left|v^{\varepsilon}-\left(u^{\varepsilon}\right)^{-}\right|<k\right\}} \mathbf{A}^{\varepsilon}\left(t, x, u^{\varepsilon}\right) D u^{\varepsilon} \quad \text { is bounded in } \quad\left(L^{2}(Q)\right)^{N}
$$

uniformly in $\varepsilon$.

Step 2. Pointwise convergence of $u^{\varepsilon}$.

Loosely speaking we consider separately the subset $u^{\varepsilon}<m$ for which we use the equation (2.16) and the subset $u^{\varepsilon} \geq m$ for which we use estimate (2.23). 
Let us first consider in $(2.16)$ a function $S \in W^{2, \infty}(\mathbb{R})$ such that $\operatorname{supp}\left(S^{\prime}\right)$ is compact in $(-\infty, m)$. Then due to $(2.21)$, we have

$$
S\left(u^{\varepsilon}\right) \text { is bounded in } L^{2}\left(0, T ; H_{0}^{1}(\Omega)\right),
$$

and

$$
\frac{\partial S\left(u^{\varepsilon}\right)}{\partial t} \text { is bounded in } L^{2}\left(0, T ; H^{-1}(\Omega)\right)+L^{1}(Q),
$$

uniformly with respect to $\varepsilon$. With a classical argument relying on an Aubin's type Lemma (see e.g. [16] and [4]) it follows that there exists a subsequence of $u^{\varepsilon}$, still indexed by $\varepsilon$, such that for any $m>\delta>0$

$$
\begin{gathered}
T_{m-\delta}^{+}\left(u^{\varepsilon}\right) \rightarrow \varphi_{\delta} \quad \text { a.e. in } Q, \\
\left(u^{\varepsilon}\right)^{-} \rightarrow \varphi^{-} \quad \text { a.e. in } Q,
\end{gathered}
$$

as $\varepsilon$ tends to 0 , where $\varphi_{\delta}$ is a nonnegative measurable function defined on $Q$ with $\varphi_{\delta} \leq m-\delta$ in $Q$ for any $\delta$ and $\varphi^{-}$is a non negative measurable function defined on $Q$.

Now remark that the sequence $\varphi_{\delta}$ is decreasing with respect to $\delta$ so that there exists a positive measurable function $\varphi^{+}$defined on $Q$ such that $\varphi^{+} \leq m$ a.e. in $Q$ and

$$
\varphi_{\delta} \rightarrow \varphi^{+} \text {a.e. in } Q
$$

as $\delta$ tends to 0 . Moreover, because of (2.35),

$$
T_{m-\delta}^{+}\left(\varphi^{+}\right)=\varphi_{\delta}
$$

for any $m>\delta>0$ so that

$$
T_{m-\delta}^{+}\left(u^{\varepsilon}\right) \rightarrow T_{m-\delta}^{+}\left(\varphi^{+}\right) \quad \text { a.e. in } Q
$$

for fixed $m>\delta>0$ as $\varepsilon$ tends to 0 . Indeed (2.39) implies that

$$
\left(u^{\varepsilon}\right)^{+} \rightarrow \varphi^{+} \quad \text { a.e. in }\left\{(t, x) \in Q ; \varphi^{+}<m\right\} .
$$

To prove that the above pointwise convergence also holds true on $\left\{(t, x) \in Q ; \varphi^{+}=m\right\}$, we use now the estimate (2.23), which shows that, extracting another subsequence,

$$
T_{2 m}^{+}\left(u^{\varepsilon}\right)-T_{m}^{+}\left(u^{\varepsilon}\right) \rightarrow 0 \text { strongly in } L^{1}(Q) \text { and a.e. in } Q \text {. }
$$

Then, from (2.39) and (2.41), we deduce that for any $\delta>0$

$$
T_{m-\delta}^{+}\left(u^{\varepsilon}\right)+T_{2 m}^{+}\left(u^{\varepsilon}\right)-T_{m}^{+}\left(u^{\varepsilon}\right) \rightarrow T_{m-\delta}^{+}\left(\varphi^{+}\right) \text {a.e. in } Q
$$

as $\varepsilon$ tends to 0 . Since indeed $\left|r^{+}-\left(T_{m-\delta}^{+}(r)+T_{2 m}^{+}(r)-T_{m}^{+}(r)\right)\right| \leq \delta$ for $r \leq 2 m$, it follows that, from $(2.42)$

$$
\left(u^{\varepsilon}\right)^{+} \rightarrow \varphi^{+} \text {a.e. in }\left\{(t, x) \in Q ; \varphi^{+}(t, x)=m\right\},
$$

as $\varepsilon$ tends to 0

In view of (2.36), (2.40) and (2.41) we finally conclude that

$$
u^{\varepsilon} \rightarrow u \quad \text { a.e. in } Q
$$

as $\varepsilon$ tends to 0 where the measurable function $u=\varphi^{+}-\varphi^{-}$is such that

$$
u \leq m \text { a.e. in } Q \text {. }
$$


Let us point out that we do not know if the sequence $v^{\varepsilon}$ pointwise converges in $Q$ in the parabolic case (due to the lack of estimate on $\frac{\partial v^{\varepsilon}}{\partial t}$ ). But, indeed, in view of $(2.44)$, we have

$$
v^{\varepsilon} \rightarrow v=\int_{0}^{u^{+}} \gamma(s) \mathrm{d} s \quad \text { a.e. in }\{(t, x) \in Q ; u(t, x)<m\},
$$

as $\varepsilon$ goes to 0 .

Step 3. Weak convergences of the fields

Upon extracting another subsequence, (2.21) and (2.44) give

$$
D T_{k}\left(u^{\varepsilon}\right) \longrightarrow D T_{k}(u) \text { weakly in } L^{2}\left(0, T ; H_{0}^{1}(\Omega)\right),
$$

as $\varepsilon$ tends to 0 and (2.21) also shows that $u$ is finite almost everywhere in $Q$. Now let us point out that the identification of $X_{k}$ and $\psi_{n}$ performed in the elliptic case only use the pointwise convergence of $v^{\varepsilon}$ on the subset $\{u<m\}$ (see the comment below the proof of $(1.50)-(1.51))$. Then from (2.47) and estimates (2.20) and (2.34), we deduce that for any $k \geq 0$ and any $n>\int_{0}^{m} \gamma(s) \mathrm{d} s$ (and for a subsequence)

$$
\begin{gathered}
\mathbf{A}^{\varepsilon}\left(t, x, u^{\varepsilon}\right)^{1 / 2} D T_{k}\left(u^{\varepsilon}\right) \rightarrow \mathbb{1}_{\{u<m\}} \mathbf{A}(t, x, u)^{1 / 2} D T_{k}(u) \quad \text { weakly in }\left(L^{2}(Q)\right)^{N} \\
h_{n}\left(v^{\varepsilon}-\left(u^{\varepsilon}\right)^{-}\right) \mathbf{A}^{\varepsilon}\left(t, x, u^{\varepsilon}\right) D u^{\varepsilon} \rightarrow \psi_{n} \quad \text { weakly in }\left(L^{2}(Q)\right)^{N},
\end{gathered}
$$

as $\varepsilon$ tends to 0 , where

$$
\psi_{n}=h_{n}\left(-u^{-}\right) \mathbf{A}(t, x, u) D u \quad \text { a.e. in }\{(t, x) \in Q ; u(t, x)<m\} .
$$

Remark that for any $k \geq 0$

$$
\mathbb{1}_{\{-k<u<m\}} \mathbf{A}(t, x, u) D u \in\left(L^{2}(Q)\right)^{N} .
$$

Step 4. Strong convergence of the energy.

In this step we prove that the convergence in $(2.48)$ is actually strong in $\left(L^{2}(Q)\right)^{N}$. We will use the technique developed by the first author and A. Porretta to deal with Stefan's type problems (see [5]). This method is simpler that the widely used on which relies on a particular time regularization introduced in [11] and adapted to renormalized solutions in [4].

In this subsection $\xi$ denotes a function in $\mathcal{C}_{0}^{\infty}([0, T))$ such that $0 \leq \xi \leq 1$.

We first choose $S^{\prime}(r)=h_{n}(r) T_{k}(r)$ and $\varphi=\xi$ in (2.16) to obtain for $k<n$

$$
\begin{aligned}
\int_{Q} \mathbf{A}^{\varepsilon}\left(t, x, u^{\varepsilon}\right) D u^{\varepsilon} \cdot D T_{k}\left(u^{\varepsilon}\right) \xi \mathrm{d} x \mathrm{~d} t & \leq \int_{Q} f h_{n}\left(u^{\varepsilon}\right) T_{k}\left(u^{\varepsilon}\right) \xi \mathrm{d} x \mathrm{~d} t \\
+\int_{Q} \xi_{t} \int_{0}^{u^{\varepsilon}} h_{n}(s) T_{k}(s) \mathrm{d} s \mathrm{~d} x \mathrm{~d} t & +\int_{\Omega} \xi(0) \int_{0}^{u_{0}} h_{n}(s) T_{k}(s) \mathrm{d} s \mathrm{~d} x \\
& +\frac{k}{n} \int_{\left\{n<\left|u^{\varepsilon}\right|<2 n\right\}} \mathbf{A}^{\varepsilon}\left(t, x, u^{\varepsilon}\right) D u^{\varepsilon} \cdot D u^{\varepsilon} \mathrm{d} x \mathrm{~d} t .
\end{aligned}
$$


We pass to the limit $\sup$ as $\varepsilon$ tends to 0 in (2.52) for fixed $n$ and $k$. In view of (2.44), this gives

$$
\begin{aligned}
\limsup _{\varepsilon \rightarrow 0} \int_{Q} \mathbf{A}^{\varepsilon}\left(t, x, u^{\varepsilon}\right) D u^{\varepsilon} \cdot D T_{k}\left(u^{\varepsilon}\right) \xi \mathrm{d} x \mathrm{~d} t & \leq \int_{Q} f h_{n}(u) T_{k}(u) \xi \mathrm{d} x \mathrm{~d} t \\
& +\int_{Q} \xi_{t} \int_{0}^{u} h_{n}(s) T_{k}(s) \mathrm{d} s \mathrm{~d} x \mathrm{~d} t+\int_{\Omega} \xi(0) \int_{0}^{u_{0}} h_{n}(s) T_{k}(s) \mathrm{d} s \mathrm{~d} x \\
& +k \limsup _{\varepsilon \rightarrow 0} \frac{1}{n} \int_{\left\{n<\left|u^{\varepsilon}\right|<2 n\right\}} \mathbf{A}^{\varepsilon}\left(t, x, u^{\varepsilon}\right) D u^{\varepsilon} \cdot D u^{\varepsilon} \mathrm{d} x \mathrm{~d} t .
\end{aligned}
$$

In view of (2.33) and since $u$ is finite almost everywhere in $Q$, we have

$$
\lim _{n \rightarrow+\infty} \limsup _{\varepsilon \rightarrow 0} \frac{1}{n} \int_{\left\{n<\left|u^{\varepsilon}\right|<2 n\right\}} \mathbf{A}^{\varepsilon}\left(t, x, u^{\varepsilon}\right) D u^{\varepsilon} \cdot D u^{\varepsilon} \mathrm{d} x \mathrm{~d} t=0 .
$$

Using the fact that $u \in L^{\infty}\left(0, T ; L^{1}(\Omega)\right),(2.15)$ and $(2.53)$, we pass to the limit as $n$ tends to $+\infty$ and we obtain

(2.54) $\limsup _{\varepsilon \rightarrow 0} \int_{Q} \mathbf{A}^{\varepsilon}\left(t, x, u^{\varepsilon}\right) D u^{\varepsilon} \cdot D T_{k}\left(u^{\varepsilon}\right) \xi \mathrm{d} x \mathrm{~d} t \leq \int_{Q} f T_{k}(u) \xi \mathrm{d} x \mathrm{~d} t$

$$
+\int_{Q} \xi_{t} \int_{0}^{u} T_{k}(s) \mathrm{d} s \mathrm{~d} x \mathrm{~d} t+\int_{\Omega} \xi(0) \int_{0}^{u_{0}} T_{k}(s) \mathrm{d} s \mathrm{~d} x .
$$

Now we use $S^{\prime}(r)=h_{n}\left(G^{\varepsilon}\left(r^{+}\right)-r^{-}\right)$in $(2.16)\left(G^{\varepsilon}\right.$ is defined in (1.28)) and this leads to

$$
\begin{aligned}
& -\|\varphi\|_{L^{\infty}(Q)} \frac{1}{n} \int_{\left\{n<\left|v^{\varepsilon}-\left(u^{\varepsilon}\right)^{-}\right|<2 n\right\}} \mathbf{A}^{\varepsilon}\left(t, x, u^{\varepsilon}\right) D u^{\varepsilon} \cdot D\left(v^{\varepsilon}-\left(u^{\varepsilon}\right)^{-}\right) \mathrm{d} x \mathrm{~d} t \\
& \leq-\int_{Q} \varphi_{t} \int_{0}^{u^{\varepsilon}} h_{n}\left(G^{\varepsilon}(s)-s^{-}\right) \mathrm{d} s \mathrm{~d} x \mathrm{~d} t-\int_{Q} \varphi(0) \int_{0}^{u_{0}} h_{n}\left(G^{\varepsilon}(s)-s^{-}\right) \mathrm{d} s \mathrm{~d} x \\
& +\int_{Q} \mathbf{A}^{\varepsilon}\left(t, x, u^{\varepsilon}\right) D u^{\varepsilon} \cdot D \varphi h_{n}\left(v^{\varepsilon}-\left(u^{\varepsilon}\right)^{-}\right) \mathrm{d} x \mathrm{~d} t-\int_{Q} f h_{n}\left(v^{\varepsilon}-\left(u^{\varepsilon}\right)^{-}\right) \varphi \mathrm{d} x \mathrm{~d} t \\
& \leq\|\varphi\|_{L^{\infty}(Q)} \frac{1}{n} \int_{\left\{n<\left|v^{\varepsilon}-\left(u^{\varepsilon}\right)^{-}\right|<2 n\right\}} \mathbf{A}^{\varepsilon}\left(t, x, u^{\varepsilon}\right) D u^{\varepsilon} \cdot D\left(v^{\varepsilon}-\left(u^{\varepsilon}\right)^{-}\right) \mathrm{d} x \mathrm{~d} t .
\end{aligned}
$$

We pass to the limit as $\varepsilon$ tends to 0 in (2.55) for fixed $n$. To this end, first remark that for $n>\int_{0}^{m} \gamma(s) \mathrm{d} s$

$$
h_{n}\left(G^{\varepsilon}(s)-s^{-}\right) \rightarrow h_{n}\left(-s^{-}\right) \mathbb{1}_{\{s<0\}}+h_{n}\left(s^{+}\right) \mathbb{1}_{\{0 \leq s \leq m\}}
$$

as $\varepsilon$ tends to 0 . As a consequence of (2.44), it follows that

$$
\int_{Q} \varphi_{t} \int_{0}^{u^{\varepsilon}} h_{n}\left(G^{\varepsilon}(s)-s^{-}\right) \mathrm{d} s \mathrm{~d} x \mathrm{~d} t \rightarrow \int_{Q} \varphi_{t}\left[\int_{0}^{-u^{-}} h_{n}(s) \mathrm{d} s+T_{m}^{+}(u)\right] \mathrm{d} x \mathrm{~d} t
$$

and

$$
\int_{\Omega} \varphi(0) \int_{0}^{u_{0}} h_{n}\left(G^{\varepsilon}(s)-s^{-}\right) \mathrm{d} s \mathrm{~d} x \rightarrow \int_{\Omega} \varphi(0)\left[\int_{0}^{-u_{0}^{-}} h_{n}(s) \mathrm{d} s+T_{m}^{+}\left(u_{0}\right)\right] \mathrm{d} x .
$$

Secondly, with the help of (2.49) and (2.50)

$$
\int_{Q} \mathbf{A}^{\varepsilon}\left(t, x, u^{\varepsilon}\right) D u^{\varepsilon} \cdot D \varphi h_{n}\left(v^{\varepsilon}-\left(u^{\varepsilon}\right)^{-}\right) \mathrm{d} x \mathrm{~d} t \rightarrow \int_{Q} \psi_{n} D \varphi \mathrm{d} x \mathrm{~d} t .
$$


At last, remark that in contrast with the elliptic case, we dot not know here that the sequence $v^{\varepsilon}$ converges pointwise on the whole set $Q$. In order to control the term $\int_{Q} f h_{n}\left(v^{\varepsilon}-\right.$ $\left.\left(u^{\varepsilon}\right)^{-}\right) \varphi \mathrm{d} x \mathrm{~d} t$, we will use (2.28) and the inequalities

$$
\begin{aligned}
\int_{Q} f \varphi \mathrm{d} x \mathrm{~d} t-\|\varphi\|_{L^{\infty}(Q)} & \int_{\left\{\left|v^{\varepsilon}-\left(u^{\varepsilon}\right)^{-}\right|>n\right\}}|f| \mathrm{d} x \mathrm{~d} t \\
\leq & \leq \int_{Q} f h_{n}\left(v^{\varepsilon}-\left(u^{\varepsilon}\right)^{-}\right) \varphi \mathrm{d} x \mathrm{~d} t \\
& \quad \leq \int_{Q} f \varphi \mathrm{d} x \mathrm{~d} t+\|\varphi\|_{L^{\infty}(Q)} \int_{\left\{\left|v^{\varepsilon}-\left(u^{\varepsilon}\right)^{-}\right|>n\right\}}|f| \mathrm{d} x \mathrm{~d} t .
\end{aligned}
$$

Setting

$$
\omega_{1}(n)=\frac{1}{n} \sup _{\varepsilon} \int_{\left\{n<\left|v^{\varepsilon}-\left(u^{\varepsilon}\right)^{-}\right|<2 n\right\}} \mathbf{A}^{\varepsilon}\left(t, x, u^{\varepsilon}\right) D u^{\varepsilon} \cdot D\left(v^{\varepsilon}-\left(u^{\varepsilon}\right)^{-}\right) \mathrm{d} x \mathrm{~d} t
$$

and

$$
\omega_{2}(n)=\sup _{\varepsilon} \int_{\left\{\left|v^{\varepsilon}-\left(u^{\varepsilon}\right)^{-}\right|>n\right\}}|f| \mathrm{d} x \mathrm{~d} t
$$

and with the help of (2.45), (2.56), (2.57) and (2.58), we pass to the limit in (2.55) as $\varepsilon$ tends to 0 and it gives

$$
\begin{gathered}
(2.62)-\|\varphi\|_{L^{\infty}(Q)}\left(\omega_{1}(n)+\omega_{2}(n)\right) \leq-\int_{Q} \varphi_{t}\left[\int_{0}^{-u^{-}} h_{n}(s) \mathrm{d} s+T_{m}^{+}(u)\right] \mathrm{d} x \mathrm{~d} t \\
-\int_{\Omega} \varphi(0)\left[\int_{0}^{-u_{0}^{-}} h_{n}(s) \mathrm{d} s+T_{m}^{+}\left(u_{0}\right)\right] \mathrm{d} x+\int_{\{u(t, x)<m\}} h_{n}\left(v-u^{-}\right) \mathbf{A}(t, x, u) D u \cdot D \varphi \mathrm{d} x \mathrm{~d} t \\
+\int_{\{u(t, x)=m\}} \psi_{n} D \varphi \mathrm{d} x \mathrm{~d} t-\int_{Q} f \varphi \mathrm{d} x \mathrm{~d} t \\
\leq\|\varphi\|_{L^{\infty}(Q)}\left(\omega_{1}(n)+\omega_{2}(n)\right) .
\end{gathered}
$$

Now we choose for the test function $\varphi$ the time regularization that is introduced in [5]. Let $u_{0 j}$ be a sequence of $\mathcal{C}_{0}^{\infty}(\Omega)$ which converges strongly to $u_{0}$ in $L^{1}(\Omega)$ and set $u(t)=u_{0 j}$ for $t<0$. We set

$$
\varphi=\xi \frac{1}{h} \int_{t-h}^{t} T_{k}(u(\tau)) \mathrm{d} \tau
$$


in (2.62). Indeed we have $\varphi \in L^{2}\left(0, T ; H_{0}^{1}(\Omega)\right) \cap L^{\infty}(Q), \varphi_{t} \in L^{\infty}(Q)$ and $\|\varphi\|_{L^{\infty}(Q)} \leq k$ (because $0 \leq \xi \leq 1$ ). It gives (recall that $\xi \in \mathcal{C}_{0}^{\infty}([0, T)$ ))

$$
\begin{gathered}
-k\left(\omega_{1}(n)+\omega_{2}(n)\right) \\
\leq-\int_{Q} \frac{\partial}{\partial t}\left(\xi \frac{1}{h} \int_{t-h}^{t} T_{k}(u(\tau)) \mathrm{d} \tau\right)\left[\int_{0}^{-u^{-}} h_{n}(s) \mathrm{d} s+T_{m}^{+}(u)\right] \mathrm{d} x \mathrm{~d} t \\
-\int_{\Omega} \varphi(0)\left[\int_{0}^{-u_{0}^{-}} h_{n}(s) \mathrm{d} s+T_{m}^{+}\left(u_{0}\right)\right] \mathrm{d} x \\
+\int_{\{u(t, x)<m\}} \xi h_{n}\left(v-u^{-}\right) \mathbf{A}(t, x, u) D u \cdot D\left[\frac{1}{h} \int_{t-h}^{t} T_{k}(u(\tau)) \mathrm{d} \tau\right] \mathrm{d} x \mathrm{~d} t \\
+\int_{\{u(t, x)=m\}} \xi \psi_{n} D\left[\frac{1}{h} \int_{t-h}^{t} T_{k}(u(\tau)) \mathrm{d} \tau\right] \mathrm{d} x \mathrm{~d} t \\
-\int_{Q} f \xi \frac{1}{h} \int_{t-h}^{t} T_{k}(u(\tau)) \mathrm{d} \tau \mathrm{d} x \mathrm{~d} t \leq k\left(\omega_{1}(n)+\omega_{2}(n)\right) .
\end{gathered}
$$

In order to deal with the parabolic contribution in (2.62), we now apply Lemma 2.3 (p. 388) of [5] with $w=u, B(r)=\int_{0}^{-r^{-}} h_{n}(s) \mathrm{d} s+T_{m}^{+}(r), \beta=B(u), \beta_{0}=B\left(u_{0}\right), w_{0}=u_{0 j}$ and $F(\lambda)=T_{k}(\lambda)$. It gives

$$
\begin{gathered}
(2.64)-\int_{Q} \frac{\partial}{\partial t}\left(\xi \frac{1}{h} \int_{t-h}^{t} T_{k}(u(\tau)) \mathrm{d} \tau\right)\left[\int_{0}^{-u^{-}} h_{n}(s) \mathrm{d} s+T_{m}^{+}(u)\right] \mathrm{d} x \mathrm{~d} t \\
-\int_{\Omega} \varphi(0)\left[\int_{0}^{-u_{0}^{-}} h_{n}(s) \mathrm{d} s+T_{m}^{+}\left(u_{0}\right)\right] \mathrm{d} x \\
\leq-\int_{Q} \xi_{t}\left[\left(\int_{0}^{-u^{-}} h_{n}(s) \mathrm{d} s+T_{m}^{+}(u)\right) \frac{1}{h} \int_{t-h}^{t} T_{k}(u(\tau)) \mathrm{d} \tau\right. \\
\left.-\frac{1}{h} \int_{t-h}^{t}\left(\int_{0}^{u(\tau)} T_{k}^{\prime}(r)\left(\int_{0}^{-r^{-}} h_{n}(s) \mathrm{d} s+T_{m}^{+}(r)\right) \mathrm{d} r \mathrm{~d} \tau\right)\right] \mathrm{d} x \mathrm{~d} t \\
-\int_{\Omega} \xi(0)\left[\left(\int_{0}^{-u_{0}^{-}} h_{n}(s) \mathrm{d} s+T_{m}^{+}\left(u_{0}\right)\right) T_{k}\left(u_{0 j}\right)-\int_{0}^{u_{0 j}} T_{k}^{\prime}(r)\left(\int_{0}^{-r^{-}} h_{n}(s) \mathrm{d} s+T_{m}^{+}(r)\right) \mathrm{d} r\right] \mathrm{d} x .
\end{gathered}
$$


Remark that, $\operatorname{since} \operatorname{supp}\left(T_{k}^{\prime}\right) \subset[-k, k]$, for $n$ large enough $\int_{0}^{r} T_{k}^{\prime}(s) \int_{0}^{-r^{-}} h_{n}(s) \mathrm{d} s=-\int_{0}^{r} T_{k}^{\prime}(s) s^{-} \mathrm{d} s$. Then letting $h$ tend to 0 in (2.64) leads to

$$
\begin{gathered}
\limsup _{h \rightarrow 0}\left(-\int_{Q} \frac{\partial}{\partial t}\left(\xi \frac{1}{h} \int_{t-h}^{t} T_{k}(u(\tau)) \mathrm{d} \tau\right)\left[\int_{0}^{-u^{-}} h_{n}(s) \mathrm{d} s+T_{m}^{+}(u)\right] \mathrm{d} x \mathrm{~d} t\right. \\
\left.-\int_{\Omega} \varphi(0)\left[\int_{0}^{-u_{0}^{-}} h_{n}(s) \mathrm{d} s+T_{m}^{+}\left(u_{0}\right)\right] \mathrm{d} x\right) \\
\leq-\int_{Q} \xi_{t}\left[\left(\int_{0}^{-u^{-}} h_{n}(s) \mathrm{d} s+T_{m}^{+}(u)\right) T_{k}(u)-\int_{0}^{u} T_{k}^{\prime}(r)\left(-r^{-}+T_{m}^{+}(r)\right) \mathrm{d} r\right] \mathrm{d} x \mathrm{~d} t \\
-\int_{\Omega} \xi(0)\left[\left(\int_{0}^{u_{0}^{-}} h_{n}(s) \mathrm{d} s+T_{m}^{+}\left(u_{0}\right)\right) T_{k}\left(u_{0 j}\right)-\int_{0}^{u_{0 j}} T_{k}^{\prime}(r)\left(-r^{-}+T_{m}^{+}(r)\right) \mathrm{d} r\right] \mathrm{d} x .
\end{gathered}
$$

Now to pass to the limit in (2.63) as $h$ tends to 0 and $j$ tend to $+\infty$, we use the fact that

$$
\frac{1}{h} \int_{t-h}^{h} T_{k}(u(\tau)) \mathrm{d} \tau \longrightarrow T_{k}(u) \quad \text { strongly in } L^{2}\left(0, T ; H_{0}^{1}(\Omega)\right)
$$

and the inequality (2.65), it yields

$$
\begin{gathered}
-k\left(\omega_{1}(n)+\omega_{2}(n)\right) \\
\leq-\int_{Q} \xi_{t}\left[\left(\int_{0}^{-u^{-}} h_{n}(s) \mathrm{d} s+T_{m}^{+}(u)\right) T_{k}(u)-\int_{0}^{u} T_{k}^{\prime}(r)\left(-r^{-}+T_{m}^{+}(r)\right) \mathrm{d} r\right] \mathrm{d} x \mathrm{~d} t \\
-\int_{\Omega} \xi(0)\left[\left(\int_{0}^{u_{0}^{-}} h_{n}(s) \mathrm{d} s+T_{m}^{+}\left(u_{0}\right)\right) T_{k}\left(u_{0}\right)-\int_{0}^{u_{0}} T_{k}^{\prime}(r)\left(-r^{-}+T_{m}^{+}(r)\right) \mathrm{d} r\right] \mathrm{d} x \\
+\int_{\{u(t, x)<m\}} \xi h_{n}\left(v-u^{-}\right) \mathbf{A}(t, x, u) D u \cdot D T_{k}(u) \mathrm{d} x \mathrm{~d} t \\
\quad+\int_{\{u(t, x)=m\}} \xi \psi_{n} \cdot D T_{k}(u) \mathrm{d} x \mathrm{~d} t-\int_{Q} f \xi T_{k}(u) \mathrm{d} x \mathrm{~d} t .
\end{gathered}
$$

Remark that $\psi_{n} \mathbb{1}_{\{u=m\}} D T_{k}(u)=0$ almost everywhere in $Q$ so that the forth term in the right hand side of (2.66) is equal to 0 . In order to pass to the limit in (2.66) as $n$ tends to $+\infty$, we first recall $(2.28)$ and $(2.32)$ so that $\omega_{1}(n) \rightarrow 0$ and $\omega_{2}(n) \rightarrow 0$ as $n$ tends to infinity (because $\left.f \in L^{1}(Q)\right)$. Secondly we use $h_{n}(r) \rightarrow 1$ for any $r$ and thirdly the fact that on the subset $\{u(t, x)<m\}$, one has $0 \leq v=\int_{0}^{u^{+}} \gamma(s) \mathrm{d} s<\int_{0}^{m} \gamma(s) \mathrm{d} s$ so that $h_{n}\left(v-u^{-}\right)=h_{n}\left(-u^{-}\right)$as soon as $n>\int_{0}^{m} \gamma(s) \mathrm{d} s$. Then we obtain

$$
\begin{aligned}
0 \leq & -\int_{Q} \xi_{t}\left[\left(-u^{-}+T_{m}^{+}(u)\right) T_{k}(u)-\int_{0}^{u} T_{k}^{\prime}(r)\left(-r^{-}+T_{m}^{+}(r)\right) \mathrm{d} r\right] \mathrm{d} x \mathrm{~d} t \\
-\int_{\Omega} \xi(0)\left[\left(-u_{0}^{-}\right.\right. & \left.\left.+T_{m}^{+}\left(u_{0}\right)\right) T_{k}\left(u_{0}\right)-\int_{0}^{u_{0}} T_{k}^{\prime}(r)\left(-r^{-}+T_{m}^{+}(r)\right) \mathrm{d} r\right] \mathrm{d} x \\
& +\int_{\{u(t, x)<m\}} \xi \mathbf{A}(t, x, u) D u \cdot D T_{k}(u) \mathrm{d} x \mathrm{~d} t-\int_{Q} f \xi T_{k}(u) \mathrm{d} x \mathrm{~d} t .
\end{aligned}
$$


Now since $u \leq m$ almost everywhere in $Q$, we have

$$
\begin{aligned}
\left(-u^{-}+T_{m}^{+}(u)\right) T_{k}(u)-\int_{0}^{u} T_{k}^{\prime}(r)\left(-r^{-}\right. & \left.+T_{m}^{+}(r)\right) \mathrm{d} r \\
& =u T_{k}(u)-\int_{0}^{u} T_{k}^{\prime}(r) r \mathrm{~d} r=\int_{0}^{u} T_{k}(r) \mathrm{d} r
\end{aligned}
$$

almost everywhere in $Q$, and the same relation holds true with $u_{0}$ in place of $u$ since $u_{0} \leq m$ also. Inserting (2.68) in (2.67) and comparing the obtained result with (2.54) yield for any $k \geq 0$

$$
\begin{aligned}
& \limsup _{\varepsilon \rightarrow 0} \int_{Q} \xi \mathbf{A}^{\varepsilon}\left(t, x, u^{\varepsilon}\right) D u^{\varepsilon} \cdot D T_{k}\left(u^{\varepsilon}\right) \mathrm{d} x \mathrm{~d} t \\
& \leq \int_{Q} \xi \mathbb{1}_{\{u<m\}} \mathbf{A}(t, x, u) D u \cdot D T_{k}(u) \mathrm{d} x \mathrm{~d} t .
\end{aligned}
$$

Then, in view of (2.48), we conclude that for any $k \geq 0$ any $0<\tau<T$

$$
\left(\mathbf{A}^{\varepsilon}\left(t, x, u^{\varepsilon}\right)\right)^{1 / 2} D T_{k}\left(u^{\varepsilon}\right) \longrightarrow \mathbb{1}_{\{u<m\}}(\mathbf{A}(t, x, u))^{1 / 2} D T_{k}(u) \quad \text { strongly in }\left(L^{2}((0, \tau) \times \Omega)\right)^{N},
$$

as $\varepsilon$ tends to 0 .

Indeed we can deduce from (2.70) (as in the elliptic case) that for any $k \geq 0$

$$
T_{k}\left(u^{\varepsilon}\right) \longrightarrow T_{k}(u) \quad \text { strongly in } L^{2}\left((0, \tau) ; H_{0}^{1}(\Omega)\right)
$$

as $\varepsilon$ tends to 0 .

Step 5. End of the proof.

Let us point out that we can not end the proof as in the elliptic case from (2.62), essentially because the function $u$ is not smooth enough with respect to $t$ to allow the choices $\varphi h(u)$ or $\varphi\left(1-b_{1 / p}\left(u^{+}\right)\right)$as test functions in $(2.62)$ which have to be in $W^{1, \infty}(Q)$. This is exactly the reason why the energy condition (2.10) is weaker than in the elliptic case.

We first prove that (2.11) holds true. To this end, consider a function $S \in \mathcal{C}^{\infty}(\mathbb{R})$ such that $S^{\prime}$ has a compact support in $(-\infty, m)$ and denote by $k$ and $k^{\prime}$ two positive real numbers such that $k^{\prime}<m$ and $\operatorname{supp}\left(S^{\prime}\right) \subset\left(-k, k^{\prime}\right)$. For any $\varphi \in \mathcal{C}_{0}^{\infty}([0, T) \times \bar{\Omega})$ such that $S^{\prime}(0) \varphi=0$ on $(0, T) \times \partial \Omega$, by $(2.16)$, the function $u^{\varepsilon}$ satisfies

$$
\begin{aligned}
-\int_{0}^{T} \int_{\Omega} \varphi_{t} S\left(u^{\varepsilon}\right) \mathrm{d} x \mathrm{~d} t-\int_{\Omega} \varphi(0) S\left(u_{0}\right) \mathrm{d} x & \\
& +\int_{0}^{T} \int_{\Omega} S^{\prime \prime}\left(u^{\varepsilon}\right) \mathbf{A}^{\varepsilon}\left(t, x, u^{\varepsilon}\right) D u^{\varepsilon} \cdot D u^{\varepsilon} \varphi \mathrm{d} x \mathrm{~d} t \\
& +\int_{0}^{T} \int_{\Omega} S^{\prime}\left(u^{\varepsilon}\right) \mathbf{A}^{\varepsilon}\left(t, x, u^{\varepsilon}\right) D u^{\varepsilon} \cdot D \varphi \mathrm{d} x \mathrm{~d} t=\int_{0}^{T} \int_{\Omega} f S^{\prime}\left(u^{\varepsilon}\right) \varphi \mathrm{d} x \mathrm{~d} t .
\end{aligned}
$$

We pass to the limit as $\varepsilon$ tends to 0 in (2.72). Since $\operatorname{supp}\left(S^{\prime}\right) \subset\left(-k, k^{\prime}\right), u^{\varepsilon}$ can be replaced by $T_{L}\left(u^{\varepsilon}\right)$ with $L=\max \left(k, k^{\prime}\right)$ in the second and third terms of (2.72). Then, due to (2.44) and $(2.70)$,

$$
S^{\prime \prime}\left(u^{\varepsilon}\right) \mathbf{A}^{\varepsilon}\left(t, x, u^{\varepsilon}\right) D T_{L}\left(u^{\varepsilon}\right) \cdot D T_{L}\left(u^{\varepsilon}\right) \longrightarrow S^{\prime \prime}(u) \mathbf{A}(t, x, u) D T_{L}(u) \cdot D T_{L}(u)
$$


strongly in $L^{1}((0, \tau) \times \Omega)$ as $\varepsilon$ tends to 0 , for any $\tau<T$ such that $\varphi(t) \equiv 0$ for $t \geq \tau$. Now since $k^{\prime}<m$,

$$
S^{\prime}\left(u^{\varepsilon}\right) \mathbf{A}^{\varepsilon}\left(t, x, u^{\varepsilon}\right) D T_{L}\left(u^{\varepsilon}\right) \longrightarrow S^{\prime}(u) \mathbf{A}(t, x, u) D T_{L}(u)
$$

weakly in $\left(L^{2}(Q)\right)^{N}$ as $\varepsilon$ tends to 0 , because of (2.44) and (2.71). At least, $S\left(u^{\varepsilon}\right)$ strongly converges to $S(u)$ in $L^{1}(Q)$ and $S^{\prime}\left(u^{\varepsilon}\right)$ converges to $S^{\prime}(u)$ weakly-* in $L^{\infty}(Q)$, as $\varepsilon$ tends to 0 . This shows that (2.11) is satisfied for any function $S$ as above. Now, using the fact that $\mathbb{1}_{\{-k<u<m\}} \mathbf{A}(t, x, u) D u \in\left(L^{2}(Q)\right)^{N}$ (see (2.51)), a standard approximation process of the function $S$ implies that (2.11) still holds true for any $S \in W^{2, \infty}(\mathbb{R})$ such that $S^{\prime}$ has a compact support and with $S^{\prime}(r)=0, r \geq m$, or equivalently with $S^{\prime}(m)=0$ since $u \leq m$ almost everywhere in $Q$ (see the comments on definition 2.1). It remains to prove (2.9) and (2.10). Upon recalling (2.70), the proof of (2.9) is classical in view of the estimate (2.53) (remark that $\left(\mathbf{A}^{\varepsilon}\left(t, x, u^{\varepsilon}\right)\right)^{1 / 2} D T_{2 n}\left(u^{\varepsilon}\right) \rightarrow \mathbb{1}_{\{u<m\}}(\mathbf{A}(t, x, u))^{1 / 2} D T_{2 n}(u)$ weakly in $\left(L^{2}(Q)\right)^{N}$ as $\varepsilon$ tends to 0$)$. To establish (2.10), we proceed as in Step 2 of this subsection and choose $S^{\prime}(r)=h_{p}(r)\left(1-b_{1 / n}\left(r^{+}\right)\right)$(for $p, n$ integers $\geq 1$ ) in (2.16). Passing first in the limit as $p$ tends to $+\infty$ as usual, we obtain for any $\varphi \in \mathcal{C}_{0}^{\infty}\left([0, T)\right.$ ) (remark that $S^{\prime}(0)=0$ so that $\varphi$ can be independent of $x$ in (2.16)),

$$
\begin{aligned}
-\int_{Q} \varphi_{t} \int_{0}^{u^{\varepsilon}} & \left(1-b_{1 / n}\left(s^{+}\right)\right) \mathrm{d} s \\
+ & n \int_{\left\{m-2 / n<u^{\varepsilon}<m-1 / n\right\}} \varphi \mathbf{A}^{\varepsilon}\left(t, x, u^{\varepsilon}\right) D u^{\varepsilon} \cdot D u^{\varepsilon} \mathrm{d} x \mathrm{~d} t \\
& =\int_{Q} f\left(1-b_{1 / n}\left(\left(u^{\varepsilon}\right)^{+}\right)\right) \varphi \mathrm{d} x \mathrm{~d} t+\int_{\Omega} \varphi(0) \int_{0}^{u_{0}}\left(1-b_{1 / n}\left(s^{+}\right)\right) \mathrm{d} s \mathrm{~d} x .
\end{aligned}
$$

In view of (2.44) and (2.70), we pass to the limit as $\varepsilon$ tends to 0 in (2.73) to obtain

$$
\begin{array}{r}
-\int_{Q} \varphi_{t} \int_{0}^{u}\left(1-b_{1 / n}\left(s^{+}\right)\right) \mathrm{d} s+n \int_{\{m-2 / n<u<m-1 / n\}} \varphi \mathbf{A}(t, x, u) D u \cdot D u \mathrm{~d} x \mathrm{~d} t \\
=\int_{Q} f\left(1-b_{1 / n}\left(u^{+}\right)\right) \varphi \mathrm{d} x \mathrm{~d} t+\int_{\Omega} \varphi(0) \int_{0}^{u_{0}}\left(1-b_{1 / n}\left(s^{+}\right)\right) \mathrm{d} s \mathrm{~d} x .
\end{array}
$$

To pass to the limit as $n$ tends to $+\infty$ in (2.74), we just remark that since $u \leq m$ almost everywhere in $Q$ and $u_{0} \leq m$ in $\Omega$,

$$
\begin{gathered}
\int_{0}^{u}\left(1-b_{1 / n}\left(s^{+}\right)\right) \mathrm{d} s \longrightarrow 0 \quad \text { strongly in } L^{1}(Q), \\
\int_{0}^{u_{0}}\left(1-b_{1 / n}\left(s^{+}\right)\right) \mathrm{d} s \longrightarrow 0 \quad \text { strongly in } L^{1}(\Omega), \\
\left(1-b_{1 / n}\left(u^{+}\right)\right) \longrightarrow \mathbb{1}_{\{u=m\}} \quad \text { a.e. in } Q \text { and weakly-* in } L^{\infty}(Q)
\end{gathered}
$$

as $n$ tends to $+\infty$.

Then we get from (2.74)

$$
\lim _{n \rightarrow+\infty} n \int_{\{m-2 / n<u<m-1 / n\}} \varphi \mathbf{A}(t, x, u) D u \cdot D u \mathrm{~d} x \mathrm{~d} t=\int_{Q} f \mathbb{1}_{\{u=m\}} \varphi \mathrm{d} x \mathrm{~d} t,
$$

for any function $\varphi \in \mathcal{C}_{0}^{\infty}([0, T))$ and $(2.10)$ is established.

The proof of Theorem 2.3 is now complete. 


\subsection{Concluding Remarks}

The above analysis is restricted to the case where $\int_{0}^{m} \gamma(s) \mathrm{d} s<+\infty$, which indeed implies that $\int_{0}^{m} \beta(s) \mathrm{d} s<+\infty$. Let us point out that if $\int_{0}^{m} \beta(s) \mathrm{d} s=+\infty$, then the analysis is simpler because one can construct a solution $u$ of (1.1) or (2.1) (i.e. in the elliptic or parabolic case) such that $u<m$ almost everywhere. Moreover it is not necessary to introduce the specific approximation $\mathbf{A}^{\varepsilon}$ of $\mathbf{A}$ given in (1.16) and the sequences $v^{\varepsilon}$ and $w^{\varepsilon}$. Indeed, if $\mathbf{A}^{\varepsilon}(x, s)=\mathbf{A}\left(x, T_{m-1 / \varepsilon}\left(s^{+}\right)-s^{-}\right)$, the approximate problems corresponding to (1.18) or (2.12) admit at least a renormalized solution $u^{\varepsilon}$. Setting $\beta^{\varepsilon}(r)=\beta\left(T_{m-1 / \varepsilon}\left(s^{+}\right)-s^{-}\right)$and using $T_{n}\left(\int_{0}^{u^{\varepsilon}} \beta^{\varepsilon}(s) \mathrm{d} s\right)$ as a test function in these approximate problems gives that, (by the same arguments as in Step 1 of the proof of Theorem 1.4 and Step 2 of the proof of Theorem $2.3)$

$$
\sup _{\varepsilon>0} \operatorname{meas}\left\{\int_{0}^{u^{\varepsilon}} \beta^{\varepsilon}(s) \mathrm{d} s>n\right\} \rightarrow 0
$$

as $n$ tends to $+\infty$.

Now, remark that the proof of the pointwise convergence of $u^{\varepsilon}$ in Part 1 or Part 2 does not use the assumption $\int_{0}^{m} \gamma(s) \mathrm{d} s<+\infty$. Then, we still have $u^{\varepsilon} \rightarrow u$ almost everywhere and $u \leq m$. But on the set $\{u=m\}, \int_{0}^{u^{\varepsilon}} \beta^{\varepsilon}(s) \mathrm{d} s \rightarrow+\infty$ as $\varepsilon$ tends to 0 , so that in view of (2.75) we obtain meas $\{u=m\}=0$. Another difference (with the case $\int_{0}^{m} \beta(s) \mathrm{d} s<+\infty$ ) is that when $\int_{0}^{m} \beta(s) \mathrm{d} s=+\infty$, we cannot expect to have $\mathbb{1}_{\{u<m\}} \mathbf{A}(x, u) D u=\mathbf{A}(x, u) D u$ belongs to $\left(L^{2}\right)^{N}$ and then equations (1.15) and (2.11) must be written with supp $(h)$ and $\operatorname{supp}\left(S^{\prime}\right)$ compact in $(-\infty, m)$.

Let us conclude this section with a few remarks on a partial uniqueness result of a solution in the sense Definition 1.2. We prove below that if $u$ and $v$ are two solutions of (1.1) such that $\{u=m\}=\{v=m\}$ then $u=v$ (see a similar situation in [3]). We restrict our comments to the elliptic case and for non negative solutions (i.e. for $f \geq 0$ ) to focus on the use of condition (1.14) on the energy (see e.g. [15] and [2] for a few conditions on $\mathbf{A}(x, s)$ for $s \leq 0$ ). First if $u \geq 0$ is solution then because of (1.12) the function $\widetilde{\beta}(u)=\int_{0}^{u} \beta(s) \mathrm{d} s$ (defined by $\widetilde{\beta}(u)=\int_{0}^{m} \beta(s) \mathrm{d} s$ on the subset $\left.\{u=m\}\right)$ belongs to $H_{0}^{1}(\Omega)$ and $D \widetilde{\beta}(u)=\mathbb{1}_{\{u<m\}} \beta(u) D u$ almost everywhere in $\Omega$ (consider $\widetilde{\beta}\left(T_{m-\varepsilon}(u)\right) \in H_{0}^{1}(\Omega)$ and let $\varepsilon$ tends to 0 ). Secondly, if we assume that $\beta(s)=\alpha \gamma(s)$ with $\alpha>0$, the matrix $\widetilde{\mathbf{A}}(x, s)=\mathbf{A}(x, s) / \beta(s)$ is uniformly coercive and bounded because of assumptions (1.6). Equation (1.15) can be rewritten as

$$
-\operatorname{div}[h(u) \widetilde{\mathbf{A}}(x, s) D \widetilde{\beta}(u)]+h^{\prime}(u) \mathbf{A}(x, u) D u \cdot D u=h(u) f \quad \text { in } \mathcal{D}^{\prime}(\Omega) .
$$

Now let us consider two solutions $u$ and $v$ of (1.1) such that $\{u=m\}=\{v=m\}$. The usual technique to prove that $u=v$ consists in plugging the test function $\left(b_{\delta}(u)-b_{\delta}(v)\right) T_{K}(\widetilde{\beta}(u)-$ $\widetilde{\beta}(v))$ in the difference of the equation for $u$ and $v$ written as (2.76) (see (1.9) for the definition of $\left.b_{\delta}\right)$. Let us point out that since $\{u=m\}=\{v=m\}$ we have $D T_{K}(\widetilde{\beta}(u)-\widetilde{\beta}(v))=0$ almost everywhere on $\{u=m\}$ and then by condition (1.14)

$$
\lim _{n \rightarrow+\infty} n \int_{\{m-2 / n<u<m-1 / n\}} \mathbf{A}(x, u) D u \cdot D u T_{K}(\widetilde{\beta}(u)-\widetilde{\beta}(v)) \mathrm{d} x=0,
$$


with a similar equality with $v$ in place of $u$. It follows that, using $b_{\delta}(u) \rightarrow \mathbb{1}_{\{u=m\}}, b_{\delta}(v) \rightarrow$ $\mathbb{1}_{\{v=m\}}$ as $\delta$ goes to 0 and because $\{u=m\}=\{v=m\}$ that

$$
\int_{\Omega}(\widetilde{\mathbf{A}}(x, u) D \widetilde{\beta}(u)-\widetilde{\mathbf{A}}(x, v) D \widetilde{\beta}(v)) \cdot D T_{K}(\widetilde{\beta}(u)-\widetilde{\beta}(v)) \mathrm{d} x=0
$$

for any $K>0$. If we assume that the matrix field $\widetilde{\mathbf{A}}(x, s)$ is uniformly Lipschitz continuous with respect to $\widetilde{\beta}(s)$ then the standard method of [1] applies and leads to $\widetilde{\beta}(u)=\widetilde{\beta}(v)$ almost everywhere in $\Omega$. Recalling the assumption $\{u=m\}=\{v=m\}$, it follows that $u=v$ almost everywhere in $\Omega$.

\section{REFERENCES}

[1] M. Artola. Sur une classe de problèmes paraboliques quasi-linéaires. Boll. Un. Mat. Ital., 5(1):51-70, 1986.

[2] D. Blanchard, F. Désir, and O. Guibé. Quasi-linear degenerate elliptic problems with $L^{1}$ data. Nonlinear Anal., 60(3):557-587, 2005.

[3] D. Blanchard and O. Guibé. Infinite valued solutions of non-uniformly elliptic problems. Anal. Appl. (Singap.), 2(3):227-246, 2004.

[4] D. Blanchard, F. Murat, and H. Redwane. Existence and uniqueness of a renormalized solution for a fairly general class of nonlinear parabolic problems. J. Differential Equations, 177(2):331-374, 2001.

[5] D. Blanchard and A. Porretta. Stefan problems with nonlinear diffusion and convection. J. Differential Equations, 210(2):383-428, 2005.

[6] D. Blanchard and H. Redwane. Quasilinear diffusion problems with singular coefficients with respect to the unknown. Proc. Roy. Soc. Edinburgh Sect. A, 132(5):1105-1132, 2002.

[7] G. Dal Maso, F. Murat, L. Orsina, and A. Prignet. Renormalized solutions of elliptic equations with general measure data. Ann. Scuola Norm. Sup. Pisa Cl. Sci. (4), 28(4):741-808, 1999.

[8] C. García Vázquez and F. Ortegón Gallego. Sur un problème elliptique non linéaire avec diffusion singulière et second membre dans $L^{1}$. C. R. Acad. Sci. Paris Sér. I Math., 332(2):145-150, 2001.

[9] C. García Vázquez and F. Ortegón Gallego. An elliptic equation with blowing-up diffusion and data in $L^{1}$ : existence and uniqueness. Math. Models Methods Appl. Sci., 13(9):1351-1377, 2003.

[10] C. García Vázquez and F. Ortegón Gallego. On certain nonlinear parabolic equations with singular diffusion and data in $L^{1}$. Commun. Pure Appl. Anal., 4(3):589-612, 2005.

[11] R. Landes. On the existence of weak solutions for quasilinear parabolic initial-boundary value problems. Proc. Roy. Soc. Edinburgh Sect. A, 89(3-4):217-237, 1981.

[12] F. Murat. Soluciones renormalizadas de EDP elipticas non lineales. Technical Report R93023, Laboratoire d'Analyse Numérique, Paris VI, 1993. Cours à l'Université de Séville.

[13] F. Murat. Equations elliptiques non linéaires avec second membre $L^{1}$ ou mesure. In Compte Rendus du 26ème Congrès d'Analyse Numérique, les Karellis, 1994. 
[14] L. Orsina. Existence results for some elliptic equations with unbounded coefficients. Asymptot. Anal., 34(3-4):187-198, 2003.

[15] A. Porretta. Uniqueness of solutions for nonlinear elliptic Dirichlet problems with $L^{1}$ data. NoDEA Nonlinear Differential Equations Appl., 11(4):407-430, 2004.

[16] J. Simon. Compact sets in the space $L^{p}(0, T ; B)$. Ann. Mat. Pura Appl. (4), 146:65-96, 1987. 